\title{
Técnicas de análisis en estudios observacionales en ciencias del deporte
}

\section{Data analysis techniques in observational studies in sport sciences}

\section{Técnicas de ciências estudos de análise de observacionais no sporte}

\author{
M. Teresa Anguera ${ }^{1}$ y Antonio Hernández-Mendo ${ }^{2}$ \\ ${ }^{1}$ Universidad de Barcelona $y^{2}$ Universidad de Málaga
}

Resumen: En este trabajo introductorio del número monográfico Análisis de datos en estudios observacionales de Ciencias del Deporte (1): Avances tecnológicos y analíticos, se realiza - desde la lógica del método cientifico y sus distintas fases- un trazado de las diversas técnicas de análisis de uso en Metodología Observacional (MO) y su importancia en el ámbito del Deporte. Se realiza una revisión en la cual los diseńos observacionales condicionan la pauta de análisis, y que ilustraremos citando los principales trabajos que conforman cada uno de ellos. Se finaliza el trabajo con un planteamiento de los retos propuestos para el futuro: (1) completar el mapa de análisis de datos con estudios empíricos de análisis de datos hasta ahora no llevados a cabo; (2) realizar avances en las propias técnicas de análisis, poniendo en relación aquéllas que admiten complementariedad de objetivos; (3) contribuir al desarrollo tecnológico e informático, que en sí mismo es un importante incentivo para avanzar en la creación de plataformas informáticas; (4) aprovechar la importancia de la fuerza y la consistencia de los mixed-methods. Palabras-clave: Metodología observacional, diseños observacionales, naturaleza de los datos, técnicas analíticas, programas informáticos.

Abstract: In this first article of monographic number, we present an approach to data analysis techniques in Observational Methodology from the framework of scientific method and taking account of several stages. All of these are rellevant in Sport Sciences field. There is a revision of some works, and moreover we present a landscape of main challenges for the future: (1) to complete a map of data analysis techniques in empirical studies, including new possibilities; (2) to improve advances in data analysis techniques, and specifically when it implies that some aims are complementary; (3) to contribute to technologic and software development, that itself it's very rellevant, and also we should move forward the creation of computerized plattforms; (4) to take advantage of the rellevance of the power and the consistency of mixed methods

Keywords: Observational methodology, observational designs, kind of data, analytic techniques, software.

Resumo: Nesta análise trabalho monografia Analise do dados introdutório em estudos observacionais de Ciências do Esporte (1): Avanços Tecnológicos e analítico, é feita-da lógica do método científico e sua diferentes fases de um caminho-de várias técnicas de análise utilizadas na metodologia observacional (MO) e sua importância no campo do Esporte. A avaliação em que a condição é realizada projetos observacionais o padrão de análise, e ilustrar citando grandes obras cada formulário. O trabalho analisa uma abordagem tendo em conta os desafios para o futuro: (1) completar o mapa análise Estudos de análise de dados empíricos até aqui asseguradas para fora; (2) fazer avanços em técnicas analíticas si, relacionando aqueles que apoiam objectivos complementares; (3) contribuir desenvolvimento tecnológico e de TI, o que em si é um importante incentivo para avançar a criação de plataformas; (4) construçáo a importância da força e consistência dos múltiplos métodos.

Palavras chave: Metodologia observacional, projetos observacionais, natureza dos dados, técnicas analíticas, programas de computador.
Las Ciencias del Deporte han seguido una trayectoria imparable en la búsqueda del conocimiento científico. Este conocimiento implica objetividad, pero también propuestas renovadas de nuevas ideas, que orientan las múltiples direcciones que ofrece este ámbito de conocimiento.

Las Ciencias del Deporte se conforman por un amplio espectro sustantivo, basado en diferentes marcos teóricos (Williams y Straub, 1991; Weinberg \& Gould, 1995; Rubio, 2001), y desplegado en una larga lista de modalidades deportivas que responden a las diferentes taxonomías existentes.

Todo estudio empírico en Ciencias del Deporte requiere forzosamente un desarrollo metodológico, mediante el cual se ofrece la respuesta que da una parcela de la realidad a la pregunta científica planteada. No se puede perder de vista

Dirección para correspondencia [Correspondence address]: Antonio Hernández Mendo. Facultad de Psicología. Universidad de Malaga (España). E-mail: mendo@um.es este planteamiento, y habrá que garantizar, en pos de los lectores de las revistas del ámbito deportivo, que la lógica del proceso empírico se ajuste a los requerimientos del método científico, y que se active la necesaria priorización de criterios dentro de la metodología observacional (MO).

Esta lógica se puede presentar de diversas maneras, atendiendo a la vía inductiva, deductiva, o mixta del estudio; en función de la metodología seguida (observacional, selectiva, cuasiexperimental o experimental); atendiendo al carácter de estudios exploratorios o confirmatorios; especificando si se formulan hipótesis o preguntas de investigación; teniendo en cuenta si se trata de estudios cualitativos, cuantitativos, o $m i$ xed-methods; diferenciando si es un estudio de caso, de caso múltiple, o poblacional; ... Pero siempre resultará imprescindible dar a conocer cómo se ha gestionado y analizado la información extraída de la realidad, es decir, los datos recogidos.

La MO, debido a la idiosincrasía que la define es, quizás, 
las más apta para el estudio de problemas sociales cambiantes, donde los procedimientos estáticos de análisis no son suficientes. Esto convierte a la $\mathrm{MO}$ en la más idónea para el estudio del deporte, cuando el objetivo es analizarlo en su contexto y dinámica habitual (Anguera y Hernández-Mendo, 2013, 2014).

Las etapas que implica la lógica del método científico, contempladas esquemáticamente, serán las cuatro siguientes en todos los casos: Delimitación del problema, recogida y optimización de los datos, análisis de datos, e interpretación de resultados. La segunda etapa, que ha dado lugar en los últimos años a un amplio número de publicaciones dedicadas a la construcción ad hoc de instrumentos de observación, termina con el control de calidad de los datos (Blanco-Villaseńor, Castellano, Hernández-Mendo, Sánchez y Usabiaga, 2014; Castellano et al., 2000; Garay-Plaza, Hernández-Mendo y Morales-Sánchez, 2006; Hernández-Mendo, Díaz-Martínez y Morales-Sánchez, 2010; Hernández-Mendo et al., 2014; Usabiaga, Castellano, Blanco-Villaseñor, y Casamichana, 2013), y la cuarta se inicia una vez que ya disponemos de los resultados. Con ello queda delimitada la tercera etapa, que es el espacio metodológico correspondiente al análisis de datos.

Y precisamente al análisis de datos está dedicado este número monográfico, acotado a estudios observacionales en Ciencias del Deporte.

\section{Revisión sobre análisis de datos en estudios observacionales del ámbito deportivo}

En este número monográfico que aquí estamos presentando se ofrece un elenco de 16 estudios observacionales, que responden a diferentes planteamientos en cuanto al análisis de datos, haciéndose eco de la diversidad existente en la comunidad científica nacional e internacional.

Uno de los autores clásicos y de gran prestigio en metodología observacional, que fue un referente en la primera etapa de desarrollo de esta disciplina, Sackett (1983), sostenía que el abanico de posibilidades analíticas en estudios donde se había utilizado observación sistemática no debía ser distinto a los que correspondan a otros planteamientos metodológicos, siempre que se adecuen a los objetivos del estudio que se pretendan en cada caso, y a las características de los datos.

Esta afirmación, realizada hace más de tres décadas, no es en absoluto baladí, y máxime dado el incipiente desarrollo de la metodología observacional en aquellos momentos. Además, fue un importante impulso para establecer los criterios básicos por los cuales se debían regir las cadenas de decisiones relativas al análisis de datos en estudios observacionales. Veamos cómo se plasma en la actualidad.

Como cuestión inicial a dirimir, la delimitación del problema de estudio estará entroncada con el ajuste al perfil que señala la opción metodológica correspondiente, y que, para la metodología observacional, implica las cuatro características fundamentales (1) de comportamiento espontáneo o habitual, (2) contexto natural o habitual, (3) instrumentos no estándar, y (4) perceptividad (Anguera, 2003, 2010).

Una vez se ha establecido que la metodología observacional es la idónea, en todo estudio empírico, la primera decisión corresponde siempre al diseño observacional, y el trabajo de Anguera, Blanco-Villaseńor y Losada (2001) fue la primera propuesta $-y$ hasta ahora la única- que se ha presentado sobre diseños observacionales (posteriormente, en Anguera, Blanco-Villaseñor, Hernández-Mendo y Losada, 2011; donde manteniendo idéntica la estructura de los diseños observacionales, se realiza una revisión en el ámbito de estudios deportivos, a la vez que se da cuenta de su amplia aplicabilidad). De acuerdo con los trabajos citados, la intersección de los criterios idiográfico-nomotético, puntual-seguimiento, y unidimensional-multidimensional, considerados dicotómicos, ha permitido la obtención de ocho diseños observacionales (I/P/U, I/P/M, I/S/U, I/S/M, N/P/U, N/P/M, N/S/U, $\mathrm{N} / \mathrm{S} / \mathrm{M})$.

A partir del trabajo mencionado de Anguera, BlancoVillaseñor y Losada (2001) se fundamentó la propuesta - no existe noticia de que en ningún ámbito geográfico se haya planteado otra alternativa, a pesar del estudio de Lees (2002)materializada poco después en Blanco-Villaseńor, Losada y Anguera (2003), en la cual, a partir de cada uno de los ocho diseños observacionales, se presenta una lista relativa a las posibilidades analíticas (Tabla 1), y efectuada desde una perspectiva de amplio espectro. Para fundamentar la solidez de cualquier propuesta se requieren unos criterios que la justifiquen, y los que se tuvieron en cuenta derivan, de forma totalmente prioritaria, del diseño observacional específico en cada caso. 
Tabla 1. Análisis de datos previsto como desarrollo de los diseños observacionales (Blanco, Losada y Anguera, 2003, p. 120-121), que a su vez se apoya en Anguera, Blanco-Villaseńor y Losada (2001).

\begin{tabular}{|c|c|c|c|}
\hline \multicolumn{2}{|c|}{ Punctual/Idiographic/Unidimensional } & \multicolumn{2}{|c|}{ Punctual/Idiographic/Multidimensional } \\
\hline $\begin{array}{l}\text { - Descriptive statistics } \\
\text { - Ordinal correlation } \\
\text { - Chi-square } \\
\text { - First order Markov } \\
\text { chains }\end{array}$ & $\begin{array}{l}\text { - Intrasessional and } \\
\text { sequential analysis } \\
\text { - Analysis of polar } \\
\text { coordinates }\end{array}$ & $\begin{array}{l}\text {-Descriptive statistics } \\
\text { - Ordinal correlation } \\
\text { - Chi-square } \\
\text { - First order Markov } \\
\text { chains } \\
\text { - Intrasessional and } \\
\text { sequential analysis }\end{array}$ & $\begin{array}{l}\text {-Analysis of polar } \\
\text { coordinates } \\
\text { - Log-lineal analysis } \\
\text { - Intra-class correlation } \\
\text { - Logistic regression } \\
\text { - Non-parametric tests }\end{array}$ \\
\hline \multicolumn{2}{|c|}{ Punctual/Nomotetic/Unidimensional } & \multicolumn{2}{|c|}{ Punctual/Nomotetic/Multidimensional } \\
\hline $\begin{array}{l}\text { - Descriptive statistics } \\
\text { - Ordinal correlation } \\
\text { - Linear correlation } \\
\text { - Chi-square } \\
\text { - First order Markov } \\
\text { chains }\end{array}$ & $\begin{array}{l}\text {-Intrasessional and } \\
\text { sequential analysis } \\
\text { - Analysis of polar } \\
\text { coordinates } \\
\text { - Non-parametric tests }\end{array}$ & $\begin{array}{l}\text { - Descriptive statistics } \\
\text { - Ordinal correlation } \\
\text { - Linear correlation } \\
\text { - Chi-square } \\
\text { - First order Markov } \\
\text { chains } \\
\text { - Intrasessional and } \\
\text { sequential analysis }\end{array}$ & $\begin{array}{l}\text {-Analysis of polar } \\
\text { coordinates } \\
\text { - intra-class correlation } \\
\text { - Logistic regression } \\
\text { - Non-parametric tests }\end{array}$ \\
\hline \multicolumn{2}{|c|}{ Follow-up/Idiographic/Unidimensional } & \multicolumn{2}{|c|}{ Follow-up /Idiographic/Multidimensional } \\
\hline $\begin{array}{l}\text { - Descriptive statistics } \\
\text { - Ordinal correlation } \\
\text { - Chi-square } \\
\text { - First order Markov } \\
\text { chains } \\
\text { - Intrasessional and } \\
\text { sequential analysis } \\
\text { - Interssional and } \\
\text { sequential analysis } \\
\text { - Analysis of polar } \\
\text { coordinates }\end{array}$ & $\begin{array}{l}\text {-Intra-class correlation } \\
\text { - Logistic regression } \\
\text { - Panel analysis } \\
\text { - Trend analysis } \\
\text { - Temporal series } \\
\text { - Analysis of variance } \\
\text { - Analysis of variance } \\
\text { for categorical data }\end{array}$ & $\begin{array}{l}\text { - Descriptive statistics } \\
\text { - Ordinal correlation } \\
\text { - Chi-square } \\
\text { - First order Markov } \\
\text { chains } \\
\text { - Intrasessional and } \\
\text { sequential analysis } \\
\text { - Interssional and } \\
\text { sequential analysis } \\
\text { - Analysis of polar } \\
\text { coordinates }\end{array}$ & $\begin{array}{l}\text {-Multiple correlation } \\
\text { - Logistic regression } \\
\text { - Panel analysis } \\
\text { - Trend analysis } \\
\text { - Multiple temporal } \\
\text { series } \\
\text { - Multiple analysis of } \\
\text { variance } \\
\text { - Multidimensional } \\
\text { scaling } \\
\text { - Non-parametric tests }\end{array}$ \\
\hline \multicolumn{2}{|c|}{ Follow-up/Nomotetic/UnidimensionaL } & \multicolumn{2}{|c|}{ Follow-up / Nomotetic/Multidimensional } \\
\hline $\begin{array}{l}\text { - Descriptive statistics } \\
\text { - Ordinal correlation } \\
\text { - Chi-square } \\
\text { - First order Markov } \\
\text { chains } \\
\text { - Intrasessional and } \\
\text { sequential analysis } \\
\text { - Interssional and } \\
\text { sequential analysis } \\
\text { - Analysis of polar } \\
\text { coordinates }\end{array}$ & $\begin{array}{l}\text {-Intra-class correlation } \\
\text { - Logistic regression } \\
\text { - Panel analysis } \\
\text { - Trend analysis } \\
\text { - Temporal series } \\
\text { - Multiple temporal } \\
\text { series } \\
\text { - Analysis of variance } \\
\text { - Analysis of variance } \\
\text { for categorical data }\end{array}$ & $\begin{array}{l}\text { - Descriptive statistics } \\
\text { - Ordinal correlation } \\
\text { - Chi-square } \\
\text { - First order Markov } \\
\text { chains } \\
\text { - Intrasessional and } \\
\text { sequential analysis } \\
\text { - Interssional and } \\
\text { sequential analysis } \\
\text { - Analysis of polar } \\
\text { coordinates }\end{array}$ & $\begin{array}{l}\text {-Multiple correlation } \\
\text { - Logistic regression } \\
\text { - Panel analysis } \\
\text { - Trend analysis } \\
\text { - Temporal series } \\
\text { - Multiple temporal } \\
\text { series } \\
\text {-Multivariate analysis } \\
\text { of variance } \\
\text { - Multidimensional } \\
\text { scaling }\end{array}$ \\
\hline
\end{tabular}

Resulta obvio, pero no por ello dejamos de indicarlo, que en un estudio observacional puede ocurrir que, si se plantean diferentes objetivos específicos, se requieran diferentes diseńos observacionales. Lo que sí queda garantizado es que cada objetivo específico hallará una respuesta en uno, y sólo uno, de los ocho diseños observacionales.
No podemos olvidar, en este sentido, que la naturaleza de cada uno de los diseños marca un sistema de subordinación de otras decisiones, como las correspondientes a la naturaleza del instrumento de observación y tipos de datos (Bakeman, 1978; Anguera, Magnusson, y Jonsson, 2007; Anguera, 2008). En este sentido, el sistema de categorías solamente 
sería posible en diseños unidimensionales, mientras que el formato de campo o la combinación de formato de campo y sistemas de categorías serían los instrumentos de observación correspondientes a los diseños multidimensionales. De forma paralela, y siguiendo la taxonomía de Bakeman (1978), los datos secuenciales (I y III) procederían de diseńos unidimensionales, mientras que los datos concurrentes (II y IV) se obtendrían en diseños mutidimensionales.

En el momento actual, y revisando el estado de la cuestión (Anguera y Hernández-Mendo, 2013, 2014), de las publicaciones recientes de estudios observacionales en el ámbito de las Ciencias del Deporte, emerge un mapa de desarrollo creciente, aunque desigual, en cuanto a la aplicación de técnicas analíticas (en algunos deportes, como el fútbol, se han realizado recientemente, de forma convergente revisiones sistemáticas, como en Sarmento, Marcelino, Anguera, Matos, Campaniço, \& Leitâo, 2014). En la Tabla 2 se presenta una revisión de las técnicas de análisis utilizadas en estudios observacionales en Ciencias del Deporte.
Posiblemente los análisis descriptivos, y especialmente en una primera época, han constituido una técnica de análisis -especialmente endeble si no se complementa con otras técnicas de análisis- que ha dado paso a otros planteamientos más específicos y robustos. Entre éstos, la mayor frecuencia de uso ha sido, indudablemente, el análisis secuencial de retardos, que en los últimos años se está viendo rebasado por la detección de T-Patterns y el análisis de variabilidad sustentado en la teoría de la generalizabilidad.

Asimismo, empiezan a adquirir consistencia los análisis específicos para datos categóricos (log-lineal, logit, probit) y aquéllos que requieren una dicotomía o dicotomización de los comportamientos observados (regresión logística). Del mismo modo, técnicas analíticas no específicas para datos categóricos, pero que pueden utilizarse de la misma forma, como análisis de tendencias, estudios correlacionales, o series temporales. En la posición opuesta, hay técnicas analíticas de las cuales no nos consta documentación en estudios observacionales en Ciencias del Deporte (como análisis de panel).

Tabla 2. Técnicas de análisis de datos en estudios observacionales en el ámbito de las Ciencias del Deporte

\begin{tabular}{|c|c|c|c|}
\hline Técnica de análisis & Año & Autores & Ámbito sustantivo \\
\hline $\begin{array}{l}\text { Análisis canónico. Es una generalización de la regresión } \\
\text { múltiple cuando hay más de una variable criterio. Por } \\
\text { este motivo es poco utilizado en metodología observacional } \\
\text { (dado que casi nunca se plantea la existencia de variables } \\
\text { predictoras y variables criterio) }\end{array}$ & 2007 & Hauw \& Durand & Trampolín \\
\hline \multirow{7}{*}{$\begin{array}{l}\text { Análisis cualitativo. Estrategia de investigación funda- } \\
\text { mentada en una depurada y rigurosa descripción contextual } \\
\text { del evento, conducta o situación que garantice la máxima } \\
\text { objetividad en la captación de la realidad, siempre comple- } \\
\text { ja, y que preserve la espontánea continuidad temporal que } \\
\text { le es inherente, con el fin de que la correspondiente recogida } \\
\text { sistemática de datos, categóricos por naturaleza, y con inde- } \\
\text { pendencia de su orientación preferente mente idiográfica y } \\
\text { procesual, posibilite un análisis (exploratorio, de reducción } \\
\text { de datos, de toma de decisiones, evaluativo, etc.) que dé } \\
\text { lugar a la obtención de conocimiento válido con suficiente } \\
\text { potencia explicativa, acorde, en cualquier caso, con el } \\
\text { objetivo planteado y los descriptores e indicadores a los que } \\
\text { se tuviera acceso (Anguera, 1986). }\end{array}$} & 2006 & Ollis, Macpherson, \& Collins & Rugby \\
\hline & 2013 & $\begin{array}{l}\text { Sarmento, Pereira, Campaniço, An- } \\
\text { guera, \& Leitão }\end{array}$ & Fútbol \\
\hline & 2013 & $\begin{array}{l}\text { Sarmento, Pereira, Matos, Campaniço, } \\
\text { Anguera, \& Leitão }\end{array}$ & Fútbol \\
\hline & 2014 & $\begin{array}{l}\text { Sarmento, Pereira, Anguera, Campa- } \\
\text { niço, \& Leitáo }\end{array}$ & Fútbol \\
\hline & 2014 & $\begin{array}{l}\text { Morales-Sánchez, Anguera, y Pérez- } \\
\text { López }\end{array}$ & $\begin{array}{l}\text { Deportes (en organi- } \\
\text { zaciones deportivas) }\end{array}$ \\
\hline & En prensa a & $\begin{array}{l}\text { Pérez-López, Morales-Sánchez, An- } \\
\text { guera, y Hernández-Mendo }\end{array}$ & $\begin{array}{l}\text { Deportes (en organi- } \\
\text { zaciones deportivas) }\end{array}$ \\
\hline & En prensa $b$ & $\begin{array}{l}\text { Pérez-López, Morales-Sánchez, An- } \\
\text { guera, y Hernández-Mendo }\end{array}$ & $\begin{array}{l}\text { Deportes (en organi- } \\
\text { zaciones deportivas) }\end{array}$ \\
\hline \multirow{7}{*}{$\begin{array}{l}\text { Análisis de contingencia. Relación entre variables categó- } \\
\text { ricas; nos permitirá conocer si existe o no relación significa- } \\
\text { tiva entre ellas. }\end{array}$} & 2006 & Lapresa, Arana, y Garzón & Fútbol \\
\hline & 2012 & Arias Estero & Baloncesto \\
\hline & 2012 & $\begin{array}{l}\text { Buscà, Moras, Peña \& Rodríguez- } \\
\text { Jiménez }\end{array}$ & Voleibol \\
\hline & 2012 & Hileno y Buscà & Voleibol \\
\hline & 2012 & Sainz de Baranda \& López-Riquelme & Fútbol \\
\hline & 2013 & Montoya, Moras, y Anguera & Balonmano \\
\hline & 2014 & $\begin{array}{l}\text { Guzmán Luján, Calpe-Gómez, Grijal- } \\
\text { bo Santamaría, y Imfeld Burkhard }\end{array}$ & Deportes colectivos \\
\hline
\end{tabular}




\begin{tabular}{|c|c|c|c|}
\hline Técnica de análisis & Año & Autores & Ámbito sustantivo \\
\hline \multirow{11}{*}{$\begin{array}{l}\text { Análisis de coordenadas polares. En una técnica de } \\
\text { reducción drástica de datos basada en el Zsum de Cochran } \\
\text { (1954), implementada por Sackett (1980) y optimizada } \\
\text { con la "técnica genuina" por Anguera (1997). Tiene como } \\
\text { objetivo la vectorialización del comportamiento y el estable- } \\
\text { cimiento de las relaciones entre una conducta focal y el resto } \\
\text { de categorías del sistema. }\end{array}$} & 1999 & Hernández-Mendo y Anguera & Deportes de equipo \\
\hline & 2000 & Gorospe y Anguera & Tenis \\
\hline & 2002 & Castellano y Hernández Mendo & Fútbol \\
\hline & 2002 & Lago y Anguera & Fútbol \\
\hline & 2003 & Castellano y Hernández-Mendo & Fútbol \\
\hline & 2012 & Robles y Castellano & Fútbol \\
\hline & 2013 & $\begin{array}{l}\text { Robles, Castellano, Perea, Martínez- } \\
\text { Santos, \& Casamichana, }\end{array}$ & Fútbol \\
\hline & 2014 & Robles, Castellano y Perea & Fútbol \\
\hline & En prensa & $\begin{array}{l}\text { Echeazarra, Castellano, Usabiaga, y } \\
\text { Hernández-Mendo }\end{array}$ & Fútbol \\
\hline & En prensa & $\begin{array}{l}\text { López-López, Menescardi, Estevan, } \\
\text { Falcó, y Hernández-Mendo }\end{array}$ & Taewondo \\
\hline & En prensa & $\begin{array}{l}\text { Sousa, Prudente, Sequeira, López- } \\
\text { López y Hernández-Mendo }\end{array}$ & Balonmano \\
\hline \multirow{14}{*}{$\begin{array}{l}\text { Análisis de Generalizabilidad (TG). Es considerada una } \\
\text { teoria de los errores multifaceta asumiendo que cualquier } \\
\text { situación de medida posee infinitas fuentes de variación o } \\
\text { facetas (Cronbach, Gleser, Nanda y Rajaratnam, 1972). A } \\
\text { través de la TG se pueden analizar las diferentes fuentes } \\
\text { de variación que pueden estar afectando a una medida o } \\
\text { diseño de medida de origen observacional. La aplicación de } \\
\text { esta teoria permite estimar el grado de generalización de un } \\
\text { diseño de medida con respecto a las condiciones particulares } \\
\text { de un valor teórico buscado. El coeficiente de generalizabi- } \\
\text { lidad permite estimar el ajuste de la media observada a la } \\
\text { media de todas las observaciones posibles. La TG unifica las } \\
\text { definiciones de fiabilidad, validez y precisión. Estas corres- } \\
\text { ponden a un aspecto parcial de un modelo más general, que } \\
\text { considera todas aquellas fuentes de variación que afectan a } \\
\text { los resultados observados. Uno de los objetivos de la medi- } \\
\text { ción es identificar y medir los componentes de variancia que } \\
\text { aportan error a una estimación e implementar estrategias } \\
\text { que reduzcan la influencia de estas fuentes de error sobre la } \\
\text { medida }\end{array}$} & 1998 & $\begin{array}{l}\text { Blanco-Villaseñor y Hernández- } \\
\text { Mendo }\end{array}$ & Fútbol \\
\hline & 2000 & $\begin{array}{l}\text { Blanco-Villaseñor, Castellano y } \\
\text { Hernández-Mendo }\end{array}$ & Fútbol \\
\hline & 2000 & $\begin{array}{l}\text { Castellano, Hernández-Mendo, Gó- } \\
\text { mez de Segura, Fontetxa y Bueno }\end{array}$ & Fútbol \\
\hline & 2008 & $\begin{array}{l}\text { Castellano, Perea, y Hernández- } \\
\text { Mendo }\end{array}$ & Fútbol \\
\hline & 2009 & $\begin{array}{l}\text { Reina, Hernández-Mendo y Fernán- } \\
\text { dez-García }\end{array}$ & Fúbol base \\
\hline & 2009 & Cuesta-Vargas y Hernández-Mendo & Control motor \\
\hline & 2010 & $\begin{array}{l}\text { Hernández-Mendo, Díaz y Morales- } \\
\text { Sánchez }\end{array}$ & Educación Física \\
\hline & 2012 & $\begin{array}{l}\text { Casamichana, Castellano, Blanco- } \\
\text { Villaseńor y Usabiaga }\end{array}$ & Fútbol \\
\hline & 2012 & $\begin{array}{l}\text { Hernández-Mendo, Montoro, Reina } \\
\text { y Fernández-García }\end{array}$ & Voleibol \\
\hline & 2012 & Hernández-Mendo y Planchuelo & Educación Física \\
\hline & 2013 & $\begin{array}{l}\text { García García, Hernández-Mendo, } \\
\text { Serrano y Morales-Sánchez }\end{array}$ & Ciclismo \\
\hline & 2013 & $\begin{array}{l}\text { Usabiaga, Castellano, Blanco-Villase- } \\
\text { ńor y Casamichana, }\end{array}$ & Iniciación deportiva \\
\hline & 2014 & $\begin{array}{l}\text { Blanco-Villaseñor, Castellano, } \\
\text { Hernández-Mendo, Sánchez-López y } \\
\text { Usabiaga }\end{array}$ & Deportes \\
\hline & En prensa & $\begin{array}{l}\text { Camerino, Castañer, Saüch, Sánchez- } \\
\text { Algarra, y Anguera }\end{array}$ & Actividad física \\
\hline \multirow{2}{*}{$\begin{array}{l}\text { Análisis de tendencias. Permite predecir, con el consi- } \\
\text { guiente riesgo de error, la relación funcional existente entre } \\
\text { una conducta y el paso del tiempo, a lo largo de, al menos, } \\
\text { tres momentos equidistantes. }\end{array}$} & 2001 & Douda, Laparidis, \& Tokmakidis & Gimnasia artística \\
\hline & 2002 & $\begin{array}{l}\text { Al-Abood, Bennett, Moreno Hernán- } \\
\text { dez, Ashford, \& Davids }\end{array}$ & Baloncesto \\
\hline
\end{tabular}




\begin{tabular}{|c|c|c|c|}
\hline Técnica de análisis & Año & Autores & Ámbito sustantivo \\
\hline \multirow{24}{*}{$\begin{array}{l}\text { Análisis de varianza. } \\
\text { Potente técnica de análisis que se basa en la descomposición } \\
\text { de la variabilidad de los datos de una variable, con el fin de } \\
\text { conocer la incidencia de las distintas fuentes de varia- } \\
\text { ción. Es posible explicar la varianza cuando se dispone de } \\
\text { variables categóricas (Anguera, Blanco-Villaseñor, Losada, } \\
\text { y Sánchez-Algarra, 1999), que son las comúnmente utiliza- } \\
\text { das en metodología observacional. }\end{array}$} & 1992 & Eom \& Schutz & Voleibol \\
\hline & 2002 & Horn, Williams, \& Scott & Fútbol \\
\hline & 2004 & $\begin{array}{l}\text { Usabiaga, Castellano, y Blanco- } \\
\text { Villaseñor }\end{array}$ & Pelota a mano \\
\hline & 2006 & Holmes, Collins, \& Calmels & $\begin{array}{l}\text { Deportes (en } \\
\text { general) }\end{array}$ \\
\hline & 2007 & $\begin{array}{l}\text { Bouquet, Gaurier, Shipley, Tousaint, } \\
\text { \& Blandin }\end{array}$ & $\begin{array}{l}\text { Deportes (en } \\
\text { general) }\end{array}$ \\
\hline & 2007 & Clark \& Ste-Marie & Natación \\
\hline & 2008 & Anderson, Ekblm, \& Krustrup & Fútbol \\
\hline & 2008 & Lonsdale \& Tam & Baloncesto \\
\hline & 2008 & Nadeau, Godbout, \& Richard & Hockey \\
\hline & 2010 & Ford, Yates, \& Williams & Fútbol \\
\hline & 2011 & $\begin{array}{l}\text { Headrick, Davids, Renshaw, Araújo, } \\
\text { Passos, \& Fernandes }\end{array}$ & Deportes de equipo \\
\hline & 2012 & Siegle \& Lames & Fútbol \\
\hline & 2013 & $\begin{array}{l}\text { Castellano, Blanco-Villaseñor, y } \\
\text { Álvarez-Pastor }\end{array}$ & Fútbol \\
\hline & 2013 & Hernández-Mendo y Planchuelo & Educación Física \\
\hline & 2014 & $\begin{array}{l}\text { Afonso, Garganta, Mcrobert, Wi- } \\
\text { lliams, \& Mesquita }\end{array}$ & Voleibol \\
\hline & 2014 & $\begin{array}{l}\text { Barreira, Garganta, Guimarães, Ma- } \\
\text { chado, \& Anguera }\end{array}$ & Fútbol \\
\hline & 2014 & $\begin{array}{l}\text { Casamichana, Castellano, y Hernán- } \\
\text { dez-Mendo }\end{array}$ & Fútbol \\
\hline & 2014 & Lanzoni, Di Michele, \& Merni & Tenis \\
\hline & 2014 & Partington, Cushion, \& Harvey & Fútbol \\
\hline & 2014 & Ramos y Hernández-Mendo & Educación Física \\
\hline & 2014 & $\begin{array}{l}\text { Uchida, Mizuguchi, Honda, \& } \\
\text { Kanosue }\end{array}$ & Baloncesto \\
\hline & In press & $\begin{array}{l}\text { Barreira, Garganta, Castellano, Ma- } \\
\text { chado, \& Anguera }\end{array}$ & Fútbol \\
\hline & En prensa & Castellano, y Blanco-Villaseñor & Fútbol \\
\hline & En prensa & Losada, Casal y Ardá & Fútbol \\
\hline \multirow{3}{*}{$\begin{array}{l}\text { Análisis log-lineal. Relación entre múltiples variables } \\
\text { categóricas, con el fin de conocer a qué modelo se ajustan los } \\
\text { datos recogidos. }\end{array}$} & 2010 & Delextrat \& Goss-Sampson & Baloncesto \\
\hline & 2010 & Delorme, Boiche, \& Raspaud & Deportes de élite \\
\hline & En prensa & Losada, Casal y Ardá & Fútbol \\
\hline \multirow{4}{*}{$\begin{array}{l}\text { Análisis no paramétrico. Abarca diferentes tipos de con- } \\
\text { trastes estadísticos que permiten poner a prueba hipótesis no } \\
\text { referidas a parámetros poblacionales. }\end{array}$} & 1999 & Castellano y Hernández-Mendo & Fútbol \\
\hline & 2003 & Bracewell & Rugby \\
\hline & 2012 & Arias, Argudo, \& Alonso & Baloncesto \\
\hline & 2012 & $\begin{array}{l}\text { Esteves, Araújo, Davids, Vilar, Travas- } \\
\text { sos, \& Esteves }\end{array}$ & Deportes de equipo \\
\hline $\begin{array}{l}\text { Análisis multivariante. Término muy amplio que alberga } \\
\text { diferentes tipos de análisis en estudios en que se dispone de } \\
\text { variables predictoras y variables criterio. }\end{array}$ & 2000 & Hernández-Mendo y Anguera & Hockey \\
\hline
\end{tabular}




\begin{tabular}{|c|c|c|c|}
\hline Técnica de análisis & Año & Autores & Ámbito sustantivo \\
\hline \multirow{22}{*}{$\begin{array}{l}\text { Análisis secuencial de retardos. El análisis secuencial } \\
\text { de retardos es una de las modalidades de análisis secuen- } \\
\text { cial, y tiene como objetivo último la detección de patrones } \\
\text { secuenciales de conducta, lo cual se lleva a cabo mediante } \\
\text { la búsqueda de contingencias secuenciales entre categorías o } \\
\text { códigos de conducta (según que el instrumento de obser- } \\
\text { vación utilizado haya sido el sistema de categorías o los } \\
\text { formatos de campo, respectivamente). }\end{array}$} & 2001 & Hernández-Mendo y Anguera & Fútbol \\
\hline & 2002 & Ardá, Casal, y Anguera & Fútbol \\
\hline & 2002 & Hernández-Mendo y Anguera & Hockey \\
\hline & 2003 & Lago y Anguera & Fútbol \\
\hline & 2004 & $\begin{array}{l}\text { García Calvo, García Herrero, i Aniz } \\
\text { Legarra }\end{array}$ & Balonmano \\
\hline & 2005 & Salas, Hileno, Molina, y Anguera & Voleibol \\
\hline & 2005 & $\begin{array}{l}\text { Silva, Sánchez Bañuelos, Garganta, y } \\
\text { Anguera }\end{array}$ & Fútbol \\
\hline & 2007 & $\begin{array}{l}\text { Castellano, Hernández-Mendo, } \\
\text { Morales-Sánchez, \& Anguera }\end{array}$ & Fútbol \\
\hline & 2008 & Salas, Molina, y Anguera & Voleibol \\
\hline & 2009 & $\begin{array}{l}\text { Santos, Fernandez, Oliveira, Leitáo, } \\
\text { Anguera, \& Campaniço }\end{array}$ & Balonmano \\
\hline & 2010 & $\begin{array}{l}\text { Afonso, Mesquita, Marcelino y Da } \\
\text { Silva }\end{array}$ & Voleibol \\
\hline & 2010 & $\begin{array}{l}\text { Castañer, Camerino, Anguera, \& } \\
\text { Jonsson }\end{array}$ & Educación Física \\
\hline & 2010 & Iglesias, Gasset, González, y Anguera & Esgrima \\
\hline & 2010 & $\begin{array}{l}\text { Louro, Silva, Anguera, Marinho, } \\
\text { Oliveira, \& Campaniço }\end{array}$ & Natación \\
\hline & 2013 & $\begin{array}{l}\text { Barbosa, Sarmento, Anzano, Angue- } \\
\text { ra, e Campaniço }\end{array}$ & Fútbol \\
\hline & 2103 & Lapresa, Arana, Anguera, \& Garzón & Fútbol \\
\hline & 2013 & $\begin{array}{l}\text { Sarmento, Pereira, Resende, } \& \\
\text { Anguera }\end{array}$ & Fútbol \\
\hline & 2013 & $\begin{array}{l}\text { Triolet, Benguigui, Le Runigo, \& } \\
\text { Williams }\end{array}$ & Tenis \\
\hline & 2014 & $\begin{array}{l}\text { Barreira, Garganta, Castellano, Pru- } \\
\text { dente, y Anguera }\end{array}$ & Fútbol \\
\hline & 2014 & $\begin{array}{l}\text { Barreira, Garganta, Machado, \& } \\
\text { Anguera }\end{array}$ & Fútbol \\
\hline & En prensa & Planes i Anguera & Fútbol \\
\hline & En prensa & Iglesias, González, \& Anguera & Taekwondo \\
\hline \multirow{2}{*}{$\begin{array}{l}\text { Comparación de medias. Relación estadística entre grupos } \\
\text { de participantes respecto a los valores de la media aritmé- } \\
\text { tica. }\end{array}$} & 2009 & $\begin{array}{l}\text { Marcelino, Mesquita, Sampaio, y } \\
\text { Anguera }\end{array}$ & Voleibol \\
\hline & 2012 & $\begin{array}{l}\text { Fernández, Sánchez, Jiménez, Nava- } \\
\text { rro, y Anguera }\end{array}$ & $\begin{array}{l}\text { Programas de } \\
\text { intervención en } \\
\text { Educación Física }\end{array}$ \\
\hline \multirow[t]{2}{*}{$\begin{array}{l}\text { Comparación de proporciones. Relación estadística entre } \\
\text { ratios correspondientes a datos de carácter cualitativo. }\end{array}$} & 2013 & $\begin{array}{l}\text { Franco, da Costa, Castañer, Fernan- } \\
\text { des, y Anguera }\end{array}$ & Actividad física \\
\hline & 2014 & $\begin{array}{l}\text { Janssen, Cliff, Reilly, Hinkley, Jones, } \\
\text { Batterham, Ekelund, Brage, \& Okely }\end{array}$ & Actividad física \\
\hline $\begin{array}{l}\text { Comparaciones múltiples. Relación estadística entre datos } \\
\text { de diversos tipos que proceden de unas mismas poblaciones y } \\
\text { que se comparan a la vez, en lugar de realizar n compara- } \\
\text { ciones independientes. }\end{array}$ & 2014 & $\begin{array}{l}\text { Correia, Passos, Araújo, Davids, Di- } \\
\text { niz, \& Kelso }\end{array}$ & Rugby \\
\hline
\end{tabular}




\begin{tabular}{|c|c|c|c|}
\hline Técnica de análisis & Año & Autores & Ámbito sustantivo \\
\hline \multirow{3}{*}{$\begin{array}{l}\text { Correlación. Relación o dependencia entre dos variables. } \\
\text { Puede ser de muy diversos tipos, según la naturaleza de los } \\
\text { datos. }\end{array}$} & 2001 & Borrie, Jonsson \& Magnusson & Fútbol \\
\hline & 2002 & Borrie, Jonsson \& Magnusson & Fútbol \\
\hline & 2014 & $\begin{array}{l}\text { Iglesias, Rodríguez-Zamora, Clapés, } \\
\text { Barrero, Chaverri, y Rodríguez }\end{array}$ & Natación \\
\hline \multirow{23}{*}{$\begin{array}{l}\text { Detección de T-Patterns. Descubrimiento de estructuras } \\
\text { ocultas que conforman la realidad del comportamiento } \\
\text { observado, pero que no resultan directamente visibles (Mag- } \\
\text { nusson, 1996, 2000, 2005, 2006). }\end{array}$} & 2003 & Anguera \& Jonsson & Fútbol \\
\hline & 2003 & $\begin{array}{l}\text { Jonsson, Bjarkadottir, Gislason, Bo- } \\
\text { rrie, \& Magnusson }\end{array}$ & Fútbol \\
\hline & 2004 & Anguera & Fútbol \\
\hline & 2004 & $\begin{array}{l}\text { Jonsson, Blanco-Villaseñor, Losada, y } \\
\text { Anguera }\end{array}$ & Fútbol \\
\hline & 2005 & $\begin{array}{l}\text { Bloomfield, Jonsson, Polman, Houla- } \\
\text { han, \& O'Donoghue }\end{array}$ & Fútbol \\
\hline & 2006 & $\begin{array}{l}\text { Jonsson, Anguera, Blanco-Villaseñor, } \\
\text { Losada, Hernández-Mendo, Ardá, } \\
\text { Camerino, \& Castellano }\end{array}$ & Fútbol \\
\hline & 2008 & $\begin{array}{l}\text { Castañer, Torrents, Dinušová, y } \\
\text { Anguera }\end{array}$ & Expresión corporal \\
\hline & 2008 & $\begin{array}{l}\text { Torrents, Castañer, Dinušová, y } \\
\text { Anguera }\end{array}$ & Danza \\
\hline & 2009 & $\begin{array}{l}\text { Castañer, Torrents, Anguera, } \\
\text { Dinušová, \& Jonsson }\end{array}$ & Conducta motora \\
\hline & 2009 & $\begin{array}{l}\text { Fernández, Camerino, Anguera, \& } \\
\text { Jonsson }\end{array}$ & Baloncesto \\
\hline & 2010 & $\begin{array}{l}\text { Chaverri, Camerino, Anguera, } \\
\text { Blanco-Villaseñor, \& Losada }\end{array}$ & Fútbol \\
\hline & 2010 & Fernández, Camerino, \& Anguera & Baloncesto \\
\hline & 2010 & $\begin{array}{l}\text { Jonsson, Anguera, Sánchez-Algarra, } \\
\text { Oliveira, Campaniço, Castañer, To- } \\
\text { rrents, Dinusová, Chaverri, Camerino, } \\
\text { \& Magnusson }\end{array}$ & $\begin{array}{l}\text { Deportes (en } \\
\text { general) }\end{array}$ \\
\hline & 2010 & $\begin{array}{l}\text { Lapresa, Arana, Garzón, Egüén i } \\
\text { Amatria }\end{array}$ & Fútbol \\
\hline & 2010 & $\begin{array}{l}\text { Torrents, Castañer, Dinušová, \& } \\
\text { Anguera }\end{array}$ & Danza \\
\hline & 2011 & Andueza, Castañer, y Camerino & Educación física \\
\hline & 2011 & $\begin{array}{l}\text { Castañer, Camerino, Parés, \& } \\
\text { Landry }\end{array}$ & Conducta motora \\
\hline & 2011 & Garzón, Lapresa, Anguera, y Arana & Baloncesto \\
\hline & 2011a & $\begin{array}{l}\text { Gutiérrez-Santiago, Prieto, Camerino, } \\
\text { \& Anguera }\end{array}$ & Judo \\
\hline & $2011 b$ & $\begin{array}{l}\text { Gutiérrez-Santiago, Prieto, Camerino, } \\
\text { y Anguera }\end{array}$ & Judo \\
\hline & 2011 & $\begin{array}{l}\text { Lapresa, García Pascual, Arana, y } \\
\text { Garzón }\end{array}$ & Baloncesto \\
\hline & 2011 & $\begin{array}{l}\text { Lapresa, Ibáñez, Arana, Amatria, i } \\
\text { Garzón }\end{array}$ & Kárate \\
\hline & 2011 & Torrents, Castañer, \& Anguera & Danza \\
\hline
\end{tabular}




\begin{tabular}{|c|c|c|c|}
\hline Técnica de análisis & Año & Autores & Ámbito sustantivo \\
\hline & 2012 & $\begin{array}{l}\text { Camerino, Chaverri, Anguera, \& } \\
\text { Jonsson }\end{array}$ & Fútbol \\
\hline & 2012 & $\begin{array}{l}\text { Gutiérrez-Santiago, Prieto, Camerino, } \\
\text { y Anguera }\end{array}$ & Balonmano \\
\hline & 2012 & Lapresa, Aragón, i Arana & Atletismo \\
\hline & 2013 & $\begin{array}{l}\text { Gutiérrez-Santiago, Prieto, Camerino, } \\
\text { y Anguera }\end{array}$ & Judo \\
\hline & 2013 & $\begin{array}{l}\text { Lapresa, Álvarez, Arana, Garzón, \& } \\
\text { Caballero }\end{array}$ & Fútbol sala \\
\hline & 2103 & Lapresa, Arana, Anguera, \& Garzón & Fútbol \\
\hline & 2013 & $\begin{array}{l}\text { Castańer, Camerino, Anguera, \& } \\
\text { Jonsson }\end{array}$ & Educación física \\
\hline & 2013 & $\begin{array}{l}\text { Prieto, Gutiérrez-Santiago, Camerino, } \\
\text { \& Anguera }\end{array}$ & Judo \\
\hline & 2013 & $\begin{array}{l}\text { Sarmento, Barbosa, Anguera, Cam- } \\
\text { paniço, \& Leitão }\end{array}$ & Fútbol \\
\hline & 2014 & $\begin{array}{l}\text { Camerino, Prieto, Lapresa, Gutiérrez, } \\
\text { y Hileno }\end{array}$ & Judo \\
\hline & 2014 & $\begin{array}{l}\text { Escolano-Pérez, Herrero-Nivela, y } \\
\text { Echeverría-Expósito }\end{array}$ & Fútbol \\
\hline & $2014 \mathrm{a}$ & Garzón, Lapresa, Anguera, y Arana & Baloncesto \\
\hline & $2014 b$ & Garzón, Lapresa, Anguera, y Arana & Baloncesto \\
\hline & 2014 & $\begin{array}{l}\text { Lapresa, Alsasua, Arana, Anguera, y } \\
\text { Garzón }\end{array}$ & Baloncesto \\
\hline & 2014 & Saüch y Castañer & Actividad física \\
\hline & 2014 & $\begin{array}{l}\text { Zurloni, Cavalera, Diana, Elia, \& } \\
\text { Jonsson }\end{array}$ & Fútbol \\
\hline & En prensa & $\begin{array}{l}\text { Alves, Franco, Castañer, Camerino, } \\
\text { Rodrigues, y Hileno }\end{array}$ & Actividad física \\
\hline & In press & $\begin{array}{l}\text { Cavalera, Diana, Elia, Jonsson, An- } \\
\text { guera, \& Zurloni }\end{array}$ & Fútbol \\
\hline & En prensa & $\begin{array}{l}\text { Iglesias, Rodríguez-Zamora, Chaverri, } \\
\text { Rodríguez, Clapés, y Anguera }\end{array}$ & Natación \\
\hline & En prensa & $\begin{array}{l}\text { Tarragó, Iglesias, Michavila, Chaverri, } \\
\text { Ruiz, y Anguera }\end{array}$ & Esgrima \\
\hline & In press & $\begin{array}{l}\text { Torrents, Castañer, Dinušová, \& } \\
\text { Anguera }\end{array}$ & Danza \\
\hline $\begin{array}{l}\text { Diagramas de Voronoi. Análisis espacial de almacena- } \\
\text { miento de la información existente entre puntos, que utiliza } \\
\text { principalmente distancias euclideas, y que es especialmente } \\
\text { adecuada cuando se dispone de datos categóricos. }\end{array}$ & In press & $\begin{array}{l}\text { Fonseca, Lopes, Leser, Baca, \& Had- } \\
\text { jileontiadis }\end{array}$ & Fútbol sala \\
\hline \multirow{2}{*}{$\begin{array}{l}\text { Inspección visual. Examen y valoración de datos sin la utiliza- } \\
\text { ción de una técnica estadística, pero sí se cuenta con la ayuda de } \\
\text { programas informáticos que permiten mejorar la visualización. }\end{array}$} & 2006 & Smith \& Cushion & Fútbol \\
\hline & 2013 & $\begin{array}{l}\text { Hernández-Mendo, Morales-Sánchez, } \\
\text { y López-Pérez }\end{array}$ & Natación \\
\hline \multirow{2}{*}{$\begin{array}{l}\text { Minería de datos. Detección de todas las relaciones com- } \\
\text { plejas existentes entre variables que se puede extraer de un } \\
\text { conjunto amplio de datos. }\end{array}$} & 2013 & $\begin{array}{l}\text { Becerra, Reigal, Hernández-Mendo, } \\
\text { y Martín-Tamayo }\end{array}$ & $\begin{array}{l}\text { Conducta motora } \\
\text { (en general) }\end{array}$ \\
\hline & En prensa & $\begin{array}{l}\text { González-Ruiz, Gómez, Pastrana, y } \\
\text { Hernández-Mendo }\end{array}$ & $\begin{array}{l}\text { Deportes (en } \\
\text { general) }\end{array}$ \\
\hline
\end{tabular}




\begin{tabular}{|c|c|c|c|}
\hline Técnica de análisis & Año & Autores & Ámbito sustantivo \\
\hline $\begin{array}{l}\text { Ratios. Medidas de asociación entre variables de carácter } \\
\text { categórico. }\end{array}$ & 2014 & $\begin{array}{l}\text { Ingebrigtsen, Dalen, Hjelde, Brust, \& } \\
\text { WislØff }\end{array}$ & Fútbol \\
\hline \multirow{2}{*}{$\begin{array}{l}\text { Regresión. Relación entre variables predictoras y variables } \\
\text { criterio, siempre que la variable criterio sea aleatoria y se } \\
\text { suponga que su valor dependa o esté influenciado por los va- } \\
\text { lores de las variables predictoras (Sánchez-Algarra, 2006). }\end{array}$} & 2011 & Afonso y Mesquita & Voleibol \\
\hline & 2011 & Marcelino, Mesquita \& Sampaio & Voleibol \\
\hline \multirow[t]{9}{*}{$\begin{array}{l}\text { Regresión logística. Relación entre variables predictoras y } \\
\text { variables criterio cuando ésta está dicotomizada. }\end{array}$} & 2012 & $\begin{array}{l}\text { Afonso, Esteves, Araújo, Thomas y } \\
\text { Mesquita }\end{array}$ & Voleibol \\
\hline & 2012 & Costa, Afonso, Brant \& Mesquita & Voleibol \\
\hline & 2013 & Arana, Lapresa, Anguera, \& Garzón & Baloncesto \\
\hline & 2013 & $\begin{array}{l}\text { Courel, Suárez, Ortega, Piñar, \& } \\
\text { Cárdenas }\end{array}$ & Baloncesto \\
\hline & 2013 & Lorenzo, Navarro, Rivilla, \& Lorenzo & Baloncesto \\
\hline & 2013 & $\begin{array}{l}\text { Peña, Rodríguez Guerra, Buscà \& } \\
\text { Serra }\end{array}$ & Voleibol \\
\hline & 2014 & Ardá, Maneiro, Rial, Losada, y Casal & Fútbol \\
\hline & In press & Arana, Lapresa, Anguera, \& Garzón & Fútbol \\
\hline & In press & $\begin{array}{l}\text { Lapresa, Arana, Anguera, Pérez, \& } \\
\text { Amatria }\end{array}$ & Fútbol \\
\hline \multirow{3}{*}{$\begin{array}{l}\text { Series temporales. Análisis de ciclos correspondiente a } \\
\text { datos ordenados temporalmente, y recogidos con distancias } \\
\text { equidistantes. }\end{array}$} & 2012 & $\begin{array}{l}\text { Travassos, Duarte, Vilar, Davids, \& } \\
\text { Araújo }\end{array}$ & Deportes de equipo \\
\hline & 2013 & Damas \& Ferreira & Baloncesto \\
\hline & 2014 & $\begin{array}{l}\text { Araújo, Davids, Diniz, Rocha, Santos, } \\
\text { Dias, \& Fernandes }\end{array}$ & Vela \\
\hline
\end{tabular}

\section{Retos futuros}

El desarrollo y solidez alcanzado por estudios observacionales en Ciencias del Deporte exige, a nuestro entender, continuar con un avance progresivo en los próximos ańos, y dar una respuesta positiva a cuatro retos que tenemos planteados en la comunidad científica nacional e internacional:

En primer lugar, completar el mapa de análisis de datos (Tabla 1) con estudios empíricos que requieran la realización de análisis de datos hasta ahora no llevados a cabo. Todavía queda camino por recorrer.

En segundo lugar, se requiere realizar avances en las propias técnicas de análisis, poniendo en relación aquéllas que admiten alguna complementariedad de objetivos (como detección de regularidades en el comportamiento), aunque los algoritmos sean totalmente distintos, o bien incorporando elementos novedosos en el desarrollo de la técnica de análisis. Un ejemplo del primer caso se halla en Lapresa, Arana, Anguera, \& Garzón (2013), en donde se han puesto en relación los resultados del análisis secuencial de retardos (mediante el programa SDIS-GSEQ) y la detección de T-Patterns (mediante el programa THEME), habiendo reflexionado sobre ello (Anguera, 2004). Y la segunda opción mencionada se ha desarrollado en Lapresa, Anguera, Alsasua, Arana, \& Garzón, 2013), llevándose a cabo un estudio de simulación de distancias convencionales, o en Lapresa, Camerino, Cabedo, Anguera, Jonsson, y Arana (en prensa, en este número monográfico), dedicado a la degradación de T-Patterns. Todavía queda mucho camino por recorrer, y deberán dedicarse esfuerzos al análisis de satellites, T-bursts, repulsion zones, taboos, drifters, packet markers, ...).

En tercer lugar, estamos convencidos de que el desarrollo tecnológico e informático es un importante incentivo. Resulta esencial avanzar en la creación de plataformas informáticas como HOISAN (Hernández-Mendo, López-López, Castellano, Morales-Sánchez, Pastrana, 2012), LINCE (Gabín, Camerino, Anguera \& Castañer, 2012), MOTS (Castellano, Perea, Alday y Hernández-Mendo, 2008) que permitan una amplia posibilidad de importación y exportación de datos respecto a otros programas utilizados en metodología observacional (Hernández-Mendo, Castellano, Camerino, Jonsson, Blanco-Villaseñor, Lopes, y Anguera, 2014). Asimismo, saludamos positivamente todos los esfuerzos realizados en la inclusión de nuevas técnicas analíticas en programas informáticos, como el análisis de coordenadas polares mediante el programa HOISAN (Hernández-Mendo, López-López, Cas- 
tellano, Morales-Sánchez, Pastrana, 2012) o la facilitación e interconectividad con otros, p.e., SAGT para el análisis de generalizabilidad (Hernández-Mendo, Ramos-Pérez y Pastrana, 2012) con HOISAN.

$\mathrm{Y}$ en cuarto y último lugar, somos perfectamente sensibles a la fuerza y consistencia de los mixed-methods, hasta hace poco considerado como una metodología emergente, pero que ahora podemos afirmar con contundencia que está ya consolidada (Camerino, Castañer, \& Anguera, 2012; Castañer, Camerino, y Anguera, 2013; Anguera, Camerino, Castañer y Sánchez-Algarra, 2014; Sarmento, Anguera, Pereira, Marques, Campaniço, \& Leitão, 2014). En torno a los mixed-methods se han desarrollado diseños específicos (Anguera, Camerino \& Castañer, 2012), que encajan perfectamente en la propia naturaleza de la metodología observacional, dado que en su primera parte es cualitativa, mientras que es cuantitativa en la segunda (Sánchez-Algarra \& Anguera, 2013).

\section{Aplicaciones prácticas}

Las aplicaciones prácticas de este artículo son evidentes, dado que en todos los estudios empíricos que utilizan la metodología observacional en Ciencias del Deporte habrá una etapa del proceso en la cual, de acuerdo con la lógica del método científico, deberá llevarse a cabo el análisis de datos. Las preguntas a las que se enfrenta todo investigador se refieren esencialmente a cuál y a cómo. Por supuesto, la delimitación precisa de los objetivos deberá ser esencial para delimitar cuáles son los análisis de datos idóneos, sean varios o incluso un único posible. En este sentido, la decisión acerca de cuál sea la técnica analítica adecuada se puede adoptar de acuerdo con las directrices recogidas en este trabajo, que, a su vez, puede ayudar a canalizar unas bases documentales iniciales orientadas al cómo llevarlo a cabo, aunque, obviamente, requerirán un desarrollo en cada caso con el fin de adquirir la pericia necesaria en su aplicación y ejecución.

Becas, ayudas o soporte financiero: Este artículo se ha realizado con el apoyo y financiación del Grup de recerca $i$ innovació en dissenys (GRID). Tecnologia $i$ aplicació multimedia $i$ digital als dissenys observacionals. Grupo Consolidado de la Generalitat de Catalunya [Referencia: 2014 SGR 971]. Asimismo, ha recibido el apoyo del Proyecto I+D+I: Observación de la interacción en deporte y actividad física: Avances técnicos y metodológicos en registros automatizados cualitativos-cuantitativos. Secretaria de Estado de Investigación, Desarrollo e Innovación del Ministerio de Economía y Competitividad [Referencia: DEP2012-32124]. También ha recibido apoyo del grupo "Evaluación Psicosocial en Contextos Naturales: Deporte y Consumo (SEJ 444)", financiado por la Junta de Andalucía (Consejería de Innovación, Ciencia y Empresa);

\section{Referencias}

1. Afonso, J., Esteves, F., Araújo, R., Thomas, L. \& Mesquita, I. (2012). Tactical determinants of setting zone in elite men's volleyball. Journal of Sports Science and Medicine, 11(1), 64-70.

2. Afonso, J., Garganta, J., Mcrobert, A., Williams, M. \& Mesquita, I. (2014). Visual search behaviours and verbal reports during film-based and in situ representative tasks in volleyball. European Journal of Sport Science, 14(2), 177-184.

3. Afonso, J. \& Mesquita, I. (2011). Determinants of block cohesiveness and attack efficacy in highlevel women's volleyball. European Journal of Sport Science, 11(1), 69-75.

4. Afonso, J., Mesquita, I., Marcelino, R. \& Da Silva, J. A. (2010). Analysis of the setter's tactical action in high-performance women's volleyball. Kinesiology, 42(1), 82-89.

5. Al-Abood, S.A., Bennett, S.J., Moreno Hernández, F., Ashford, D., \& Davids, K. (2002). Effect of verbal instructions and image size on visual search strategies in basketball free throw shooting. Journal of Sports Sciences, 20, 271-278.

6. Alves, S., Franco, S., Castañer, M., Camerino, O., Rodrigues, J. y Hileno, R. (en prensa). El análisis de la comunicación paraverbal cinésica y proxémica de los entrenadores de fitness mediante patrones temporales (T-patterns). Cuadernos de Psicología del Deporte [en este número monográfico].

7. Anderson, H., Ekblm, B, \& Krustrup, P. (2008). Elite football on artificial turf versus natural grass: Movement patterns, technical standards, and player impressions. Journal of Sports Sciences, 26(2), 113-122.

8. Andueza, J., Castañer, M. y Camerino, O. (2011). Detección de patrones de interacción y cohesión de grupo-clase en educación física. Tándem. Didáctica de la Educación Física, 35, 75-83.
9. Anguera, M. T., Camerino, O. \& Castañer, M. (2012). Mixed methods procedures and designs for research on sport, physical education and dance. In O. Camerino, M. Castañer \& M.T. Anguera (Ed.), Mixed Methods Research in the Movement Sciences: Case studies in sport, physical education and dance (pp. 3-27). Abingdon, UK: Routledge.

10. Anguera, M.T. \& Jonsson, G.K. (2003). Detection of real time patterns in sport: Interactions in football. International Journal of Computer Science in Sport (e-Journal), 2(2), 118-121.

11. Anguera, M.T. (1986). Investigación cualitativa. Educar, 10, 23-50.

12. Anguera, M.T. (1997). From prospective patterns in behavior to joint analysis with a retrospective perspective. Colloque sur invitation "Méthodologie d'analyse des interactions sociales». Université de la Sorbonne. Paris.

13. Anguera, M.T. (2003). Observational Methods (General). In R. Fernández-Ballesteros (Ed.), Encyclopedia of Psychological Assessment, Vol. 2 (pp. 632-637). London: Sage.

14. Anguera, M.T. (2004). Hacia la búsqueda de estructuras regulares en la observación del fútbol: Detección de patrones temporales. Cultura, Ciencia y Deporte. Revista de Ciencias de la Actividad Física y del Deporte (Murcia), 1(1), 15-20.

15. Anguera, M.T. (2008). Diseños evaluativos de baja intervención. En M.T. Anguera, S. Chacón y A. Blanco-Villaseńor (Coords.), Evaluación de programas sanitarios y sociales. Abordaje metodológico (pp. 153184). Madrid: Síntesis.

16. Anguera, M.T. (2010). Posibilidades y relevancia de la observación sistemática por el profesional de la Psicología. Papeles del Psicólogo, 31(1), 122-130.

17. Anguera, M.T. y Hernández-Mendo, A. (2014). Metodología observa- 
cional y psicología del deporte. Estado de la cuestión. Revista de Psicología del Deporte, 23(1), 103-109.

18. Anguera, M.T., Blanco-Villaseñor, A. y Losada, J.L. (2001). Diseńos Observacionales, cuestión clave en el proceso de la metodología observacional. Metodología de las Ciencias del Comportamiento, 3(2), 135-161.

19. Anguera, M.T., Blanco-Villaseñor, A., Hernández-Mendo, A., y Losada, J.L. (2011). Diseños observacionales: ajuste y aplicación en psicología del deporte. Cuadernos de Psicología del Deporte, 11(2), 63-76.

20. Anguera, M.T., Blanco-Villaseñor, A., Losada, J.L. y Sánchez-Algarra, P. (1999). Análisis de la competencia en la selección de observadores. Metodología de las Ciencias del Comportamiento, 1(1), 95-114.

21. Anguera, M.T., Camerino, O., Castañer, M. y Sánchez-Algarra, P. (2014). Mixed methods en actividad física y deporte. Revista de Psicologia del Deporte, 23(1), 123-130.

22. Anguera, M.T., Magnusson, M.S. y Jonsson, G.K. (2007). Instrumentos no estándar. Avances en Medición, 5(1), 63-82.

23. Anguera, M.T., y Hernández-Mendo, A. (2013). La metodología observacional en el ámbito del deporte. E-balonmano.com: Revista de Ciencias del Deporte, 9(3), 135-160. http://www.e-balonmano.com/ ojs/index.php/revista/index

24. Arana, J., Lapresa, D., Anguera, M.T. \& Garzón, B. (2013). Adapting football to the child: an application of the logistic regression model in observational methodology. Quality \& Quantity, 47(6), 3473-3480.

25. Arana, J., Lapresa, D., Anguera, M.T. \& Garzón, B. (in press). Application of logistic regression models in observational methodology: Game formats in grassroots football in initiation into football. Anales de Psicología.

26. Araújo, D., Davids, K., Diniz, A., Rocha, L., Santos, J.C., Dias, G., \& Fernandes, O. (2014). Ecological dynamics of continuous and categorical decision-making: The regatta start in sailing. European Journal of sport Science, http://dx.doi.org/10.1080/17461391.2014.928749

27. Ardá, A., Maneiro, R., Rial, A., Losada, J.L. y Casal, C.A. (2014). Análisis de la eficacia de los saques de esquina en la Copa del Mundo de Fútbol 2010. Un intento de identificación de variables explicativas. Revista de Psicología del Deporte, 23(1), 165-172.

28. Ardá, T., Casal, C.A. y Anguera, M.T. (2002). Evaluación de las acciones ofensivas de éxito en fútbol 11 mediante diseños diacrónicos intensivos retrospectivos. Metodología de las Ciencias del Comportamiento, vol. especial, 48-51.

29. Arias Estero, J.L. (2012). Anàlisi de la situació d'un contra un en bàsquetbol de formació. Apunts. Educació Física i Esports, 107(1), 54-60.

30. Arias, J.L., Argudo, F.M., \& Alonso, J.I. (2012). Effect of the ball mass on the one-on-one game situation in 9-11 year old boys' basketball. European Journal of Sport Science, 12(3), 225-230.

31. Bakeman, R. (1978). Untangling streams of behavior: Sequential analysis of observation data. In G.P. Sackett (Ed.), Observing Behavior, Vol. 2: Data collection and analysis methods (pp. 63-78). Baltimore: University of Park Press.

32. Barbosa, A., Sarmento, H., Anzano, A., Anguera, M.T. e Campaniço, J. (2013). Estudo dos métodos de jogo ofensivo no futebol: Circunscriçấo das açôes de desenvolvimiento com a ativaçáo zonal. Revista Mineira de Educação Fisica, 9 (Edição Especial), 593-599.

33. Barreira, D., Garganta, J., Castellano, J., Prudente, J. y Anguera, M.T. (2014). Evolución del ataque en el fútbol de élite entre 1982 y 2010: Aplicación del análisis secuencial de retardos. Revista de Psicología del Deporte, 23(1), 139-146.

34. Barreira, D., Garganta, J., Guimarăes, P., Machado, J., \& Anguera, M.T. (2014). Ball recovery patterns as a performance indicator in elite soccer. Proc IMechE Part P: Journal of Sports Engineering and Technolo$g y, 228(1), 61-72$.

35. Barreira, D., Garganta, J., Machado, J.C. \& Anguera, M.T. (2014). Effects of ball recovery in top-level soccer attacking patterns of play. Revista Brassileira de Cineantropometria \& Desempenho Humano, 16(1), $36-46$.
36. Barreira, D.,Garganta, J., Castellano, J., Machado, J. \& Anguera, M.T. (in press). How elite-level soccer dynamics has evolved over the last three decades: Input from generalizability theory. Cuadernos de Psicología del Deporte [in this monographic number].

37. Becerra, C.A., Reigal, R., Hernández-Mendo, A., y Martín-Tamayo, I. (2013). Relaciones de la condición física y la composición corporal con la autopercepción de salud. Revista Internacional de Ciencias del Deporte, 34, 305-318.

38. Blanco-Villaseñor, A. y Hernández-Mendo, A. (1998). Estimación y generalización en un diseńo de estructura espacial. En J. Sabucedo, R. García, E.Ares y D. Prada, Medio ambiente y responsabilidad humana (pp. 579-583). A Coruńa: Libro de Comunicaciones. VI Congreso de Psicología Ambiental.

39. Blanco-Villaseñor, A., Castellano, J. y Hernández Mendo, A. (2000). Generalizabilidad de las observaciones en la acción del juego en el fútbol. Psicothema, suplemento, 12(Supl. 2), 81-86.

40. Blanco-Villaseñor, A., Castellano, J., Hernández-Mendo A., Sánchez, C.R. y Usabiaga, O. (2014). Aplicación de la TG en el deporte para el estudio de la validez, fiabilidad y estimación de la muestra. Revista de Psicología del Deporte, 23(1), 131-137.

41. Blanco-Villaseñor, A., Losada, J.L. y Anguera, M.T. (2003). Analytic techniques in observational designs in environment-behavior relation. Medio Ambiente y Comportamiento Humano, 4 (2), 111-126.

42. Bloomfield, J., Jonsson, G.K., Polman, R., Houlahan, K. \& O'Donoghue, P. (2005) Temporal pattern analysis and its applicability in soccer. In L. Anolli, S. Duncan, M.S. Magnusson \& G. Riva (Eds.), The hidden structure of social interaction: From genomics to culture patterns (pp. 237-251). Amsterdam: IOS Press.

43. Borrie, A., Jonsson, G.K. \& Magnusson, M.S. (2001). Application of t-pattern detection and analysis in sports research. Metodología de las Ciencias del Comportamiento, 3(2), 215-226.

44. Borrie, A., Jonsson, G.K. \& Magnusson, M.S. (2002). Temporal pattern analysis and it's applicability in sport: an explanation and preliminary data. Journal of Sport Science, 20(10), 845 - 852.

45. Bouquet, C.A., Gaurier, V., Shipley, T., Tousaint, L., \& Blandin, Y. (2007). Influence of the perception of biological or non-biological motion on movement execution. Journal of Sports Sciences, 25(5), 519-530.

46. Bracewell, P.J. (2003). Monitoring meaningful rugby ratings. Journal of Sports Sciences, 21, 611-620.

47. Buscà, B., Moras, G., Peña, J. \& Rodríguez-Jiménez, S. (2012). The influence of serve characteristics on performance in men's and women's high-standard beach volleyball. Journal of Sports Sciences, 30(3), 269276.

48. Camerino, O., Castañer, M. \& Anguera, M.T. (Coords.) (2012), Mixed Methods Research in the Movement Sciences: Case studies in sport, physical education and dance. Abingdon, UK.: Routledge.

49. Camerino, O., Castañer, M., Saüch, G., Sánchez-Algarra, P. y Anguera, M.T. (en prensa). Percepción de la intensidad al esfuerzo: Un estudio multimethod en actividad física. Cuadernos de Psicología del Deporte [en este número monográfico].

50. Camerino, O., Chaverri, J., Anguera, M.T. \& Jonsson, G. (2012). Dynamics of the game in soccer: Detection of t-patterns. European Journal of Sport Science, 12(3), 216-224.

51. Camerino, O., Prieto, I., Lapresa, D., Gutiérrez, A., y Hileno, R. (2014). Detección de T-Patterns en la observación de deportes de combate. Revista de Psicología del Deporte, 23(1), 147-155.

52. Casamichana, D., Castellano, J. y Hernández-Mendo, A. (2014). La Teoría de la Generalizabilidad aplicada al estudio del perfil físico durante juegos reducidos con diferente orientación del espacio en fútbol. Revista Internacional de Ciencias del Deporte, 10(37), 195-205.

53. Castañer, M., Camerino, O. y Anguera, M.T (2013). Métodos mixtos en la investigación de las ciencias de la actividad física y el deporte. Apunts. Educación Fisica y Deporte, 112(2), 31-36. 
54. Castañer, M., Camerino, O., Anguera, M.T \& Jonsson, G.K. (2013) Kinesics and proxemics communication of expert and novice PE teachers. Quality \& Quantity, 47(4), 1813-1829.

55. Castañer, M., Camerino, O., Anguera, M.T. \& Jonsson, G.K. (2010). Observing the paraverbal communicative style of expert and novice PE teachers by means of SOCOP: a sequential analysis. Procedia - Social and Behavioral Sciences. Innovation and reativity in Education, 2(2), 5162-5167.

56. Castañer, M., Camerino, O., Parés, N. \& Landry, L. (2011). Fostering body movement in children through an exertion interface as an educational tool. Procedia - Social and Behavioral Sciences, 28, 236 - 240.

57. Castañer, M., Torrents, C., Anguera, M.T., Dinušová, M. \& Jonsson, G. (2009). Identifying and analyzing motor skill responses in body movement and dance. Behavior Research Methods, 41(3), 857-867.

58. Castañer, M., Torrents, C., Dinušová, M., y Anguera, M.T. (2008). Habilidades motrices en expresión corporal y danza. Detección de T-Patterns. Motricidad. European Journal of Human Movement, 21, 168-188.

59. Castellano, J. y Blanco-Villaseñor, A. (en prensa). Análisis del perfil de desplazamiento de futbolistas de élite durante una temporada competitiva a partir de un modelo mixto multivariable. Cuadernos de Psicología del Deporte [en este número monográfico].

60. Castellano, J. y Hernández-Mendo, A. (1999). Análisis secuencial en el fútbol de rendimiento. Psicothema, 12 (supl. 2), 117-121.

61. Castellano, J. y Hernández-Mendo, A. (2002). Aportaciones del análisis de coordenadas polares en la descripción de las transformaciones de los contextos de interacción defensivos en Fútbol. Cronos, 1, 42-48.

62. Castellano, J. y Hernández-Mendo, A. (2003). El análisis de coordenadas polares para la estimación de relaciones en la interacción motriz en fútbol. Psicothema, 15(4), 569-574.

63. Castellano, J., Blanco-Villaseñor, A., y Álvarez-Pastor, D. (2013). Análisis del espacio de interacción en fútbol. Revista de Psicología del Deporte, 22(2), 437-446

64. Castellano, J., Hernández-Mendo, A., Gómez de Segura, P., Fontetxa, E. y Bueno, I. (2000). Sistema de codificación y análisis de la calidad del dato en el fútbol de rendimiento. Psicothema, 12(4), 636-641.

65. Castellano, J., Hernández-Mendo, A., Morales-Sánchez, V. \& Anguera, M.T. (2007). Optimizing a probabilistic model of the development of play in soccer. Quality \& Quantity. International Journal of Methodo$\log y, 41(1), 93-104$.

66. Castellano, J., Perea, A. y Hernández-Mendo, A. (2008). Análisis de la evolución del fútbol a lo largo de los mundiales. Psicothema, 20(4), 928-932.

67. Castellano, J. Perea, A., Alday, L. y Hernández-Mendo, A. (2008). The Measuring and Observation Tool in Sports. Behavior Research Methods, 40 (3), 898-905.

68. Cavalera, C., Diana, B., Elia, M., Jonsson, G., Anguera, M.T. \& Zurloni, V. (in press). Performance analysis in team sports: A T-pattern approach. Cuadernos de Psicología del Deporte [in this monographic number].

69. Chaverri, J., Camerino, O., Anguera, M.T., Blanco-Villaseñor, Á. \& Losada, J.L. (2010). Interaction contexts in soccer: Detection of TPatterns. Gymnasium. Revista de Educação Física, Desporto e Saúde, 2(1), 69-92.

70. Clark, S.E. \& Ste-Marie, D.M. (2007). The impact of self-as-a-model interventions on children's self-regulation of learning and swimming performance. Journal of Sports Sciences, 25(5), 577-586.

71. Cochran, W.G. (1954). Some methods for strengthening the common tests. Biometrics, 10, 417-451.

72. Correia, V., Passos, P., Araújo, D., Davids, K., Diniz, A., \& Kelso, J.A.S. (2014). Coupling tendencies during exploratory behaviours of competing players in rugby union dyads. European Journal of Sport Science, http://dx.doi.org/10.1080/17461391.2014.915344

73. Costa, G., Afonso, J., Brant, E. \& Mesquita, I. (2012). Differences in game patterns between male and female youth volleyball. Kinesiology, 44(1), 60-66.

74. Courel, J., Suárez, E., Ortega, E., Piñar, M. \& Cárdenas, D. (2013). Is the inside pass a performance indicator? Observational analysis of elite basketball teams. Revista de Psicología del Deporte, 22(1), 191-194.

75. Cuesta-Vargas, A. I. y Hernández-Mendo, A. (2009). Fiabilidad de un sistema de clasificación clínica de lumbalgia mecánica inespecífica para el tratamiento de la estabilidad lumbo pélvica mediante el control motor. Cuest. fisioter, 38(1):11-18.

76. Damas, R.S. \& Ferreira, A.P. (2013). Patterns of visual search in basketball coaches. An analysis on the level of performance. Revista de Psicología del Deporte, 22(1), 199-204.

77. Delextrat, A. \& Goss-Sampson, M. (2010). Kinematic analysis of netball goal shooting: A comparison of junior and senior players. Jour nal of Sports Sciences, 28(12), 1299-1307.

78. Delorme, N., Boiche, J., \& Raspaud, M. (2010). Relative age effect in elite sports: Methodological bias or real discrimination? European Journal of Sport Science, 10(2), 91-96.

79. Douda, H., Laparidis, K., \& Tokmakidis, S.P. (2002). Long-Term Training Induces Specific Adaptations on the Physique of Rhythmic Sports and Female Artistic Gymnasts. European Journal of Sport Science, 2(3), 1-14

80. Echeazarra, I., Castellano, J., Usabiaga, O. y Hernández-Mendo, A. (en prensa). Comparación del uso del espacio en categorías infantil y cadete de fútbol a partir del análisis de coordenadas polares. Cuadernos de Psicología del Deporte [en este número monográfico].

81. Eom, H. J. \& Schutz, R. W. (1992). Transition play in team performance of volleyball a log-linear analysis. Research Quarterly for Exercise and Sport, 63(3), 261-269.

82. Escolano-Pérez, E., Herrero-Nivela, M.L. y Echeverría-Expósito, R. (2014). El Descubrimiento guiado como estrategia de enseńanza para el aprendizaje de la táctica ofensiva en la categoría pre-benjamín de futbol 8. Revista de Psicología del Deporte, 23(1), 209-217.

83. Esteves, P.T., Araújo, D., Davids, K., Vilar, L., Travassos, B., \& Esteves, C. (2012). Interpersonal dynamics and relative positioning to scoring target of performers in 1 vs. 1 sub-phases of team sports. Journal of Sports Sciences, 30(12), 1285-1293.

84. Fernández, J., Camerino, O., \& Anguera, M.T. (2010) Identifying and analyzing the construction and effectiveness of offensive plays in basketball by using systematic observation. International Journal of Sport Psychology \& Coaching (special issue), 65.

85. Fernández, J., Camerino, O., Anguera, M.T. \& Jonsson, G. (2009) Identifying and analyzing the construction and effectiveness of offensive plays in basketball by using systematic observation. Behavior Research Methods, 41(3), 719-730.

86. Fernández, M., Sánchez, C.R., Jiménez, F., Navarro, V. y Anguera, M.T. (2012). Sistema de codificación y análisis de la calidad del dato para una intervención inclusiva en Educación Física. Revista de Psicología del Deporte, 21(1), 67-73.

87. Fonseca, S., Lopes, A., Leser, R., Baca, A., \& Hadjileontiadis, L. (in press). Classification power of spatial metrics. An application in futsal. Cuadernos de Psicología del Deporte [in this monographic number].

88. Ford, P.R., Yates, I., \& Williams, A.M. (2010). An analysis of practice activities and instructional behaviours used by youth soccer coaches during practice: Exploring the link between science and application. Journal of Sports Sciences, 28(5), 483-495.

89. Franco, S., da Costa, V., Castañer, M., Fernandes, J. \& Anguera, M.T. (2013). La conducta de los instructores de Fitness: triangulación entre la percepción de los practicantes, auto-percepción de los instructores y conducta observada. Revista de Psicología del Deporte, 22(2), 321-329.

90. Gabín, B., Camerino, O., Anguera, M. T. \& Castañer, M. (2012). Lince: Multiplatform sport analysis software. Procedia-Social and Behavioral Sciences, 46, 4692-4694. 
91. Garay-Plaza, J.O., Hernández-Mendo, A., y Morales-Sánchez, V. (2006). Sistema de codificación y análisis de la calidad del dato en el tenis de dobles. Revista de Psicología del Deporte, 15(2), 279-294.

92. García Calvo, T., García Herrero, J.A. i Aniz Legarra, I. (2004). Anàlisi de l'estructura de l'atac en equips d'alt nivell d'handbol. Apunts. Educació Física i Esport, 76(2), 53-68.

93. García García, O., Hernández-Mendo, A., Serrano, V. y Morales-Sánchez, V. (2013). Aplicación de la teoría generalizabilidad a un análisis de tensiomiografía en ciclistas profesionales de ruta. Revista de Psicologia del Deporte, 22(1), 53-60.

94. Garzón, B., Lapresa, D., Anguera, M.T. y Arana, J. (2011). Análisis observacional del lanzamiento de tiro libre en jugadores de baloncesto base. Psicothema, 23(4), 851-857.

95. Garzón, B., Lapresa, D., Anguera, M.T. y Arana, J. (2014a). Del minibasket al baloncesto: Efectos de la actual configuración reglamentaria en el patrón técnico de lanzamiento de tiro libre. Revista de Psicología del Deporte, 23(1), 77-85.

96. Garzón, B., Lapresa, D., Anguera, M.T. y Arana, J. (2014b). Estudio de propuestas intermedias de baloncesto en categoría infantil. Revista Iberoamericana de Ciencias de la Actividad Física y el Deporte, 1(1), 10-21.

97. González-Ruiz, S., Gómez, I., Pastrana, J. L. y Hernández-Mendo, A. (en prensa). Algoritmos de clasificación y redes neuronales en la observación automatizada de registros. Cuadernos de Psicología del Deporte [en este número monográfico].

98. Gorospe, G. y Anguera, M.T. (2000). Modificación de la técnica clásica de coordenadas polares mediante un desarrollo distinto de la retrospectividad: Aplicación al tenis. Psicothema, 12 (Supl. No 2), 279-282.

99. Gutierrez-Santiago, A., Prieto, I., Camerino, O. \& Anguera, M.T. (2013). Sequences of errors in the judo throw Morote Seoi Nage and their relationship to the learning process. Journal of Sports Engineering and Technology, 227(1), 57-63.

100. Gutiérrez-Santiago, A., Prieto, I., Camerino, O. \& Anguera, M.T. (2011a). The temporal structure of judo bouts in visually impaired men and women. Journal of Sports Sciences, 29(13), 1443-1451.

101. Gutiérrez-Santiago, A., Prieto, I., Camerino, O. y Anguera, M.T. (2011b). Identificación y análisis del aprendizaje del judo mediante la metodología observacional. Apunts. Educación Física y Deportes, $104(2), 46-55$.

102. Gutiérrez-Santiago, A., Prieto, I., Camerino, O. y Anguera, M.T. (2012). Eficacia de los sistemas ofensivos en balonmano. Apunts. Educación Física y Deportes, 108, 66-77.

103. Guzmán Luján, J.F., Calpe-Gómez, V., Grijalbo Santamaría, C. y Imfeld Burkhard, F. (2014). Una observación sistemática de las conductas verbales de los entrenadores en función de las acciones de juego competitivas. Revista de Psicología del Deporte, 23(2), 301-307.

104. Hauw, D. \& Durand, M. (2007). Situated analysis of elite trampolinists' problems in competition using retrospective interviews. Journal of Sports Sciences, 25(2), 173-183.

105. Headrick, J., Davids, K., Renshaw, I., Araújo, D., Passos, P., \& Fernandes, O. (2011). Proximity-to-goal as a constraint on patterns of behaviour in attacker-defender dyads in team games. Journal of Sports Sciences, 30(3), 247-253.

106. Hernández-Mendo A., Morales-Sánchez, V. y López-Pérez, A.A. (2013). Efectividad de una intervención psicológica con un nadador de aguas abiertas. Avances de la Psicologİa del Deporte en Iberoamérica, 2(1), 31-46.

107. Hernández-Mendo, A. \& Anguera, M.T. (2002). Behavioral structure in sociomotor sports: Roller-Hockey. Quality \& Quantity. European Journal of Methodology, 36, 347-378.

108. Hernández-Mendo, A. y Anguera, M.T. (1999). Aportaciones de análisis de coordenadas polares a los deportes de equipo. En F. Guillén (Ed.) La Psicología del Deporte en España al final del milenio (pp. 169-175). Las Palmas: Universidad de Las Palmas de Gran Canaria.
109. Hernández-Mendo, A. y Anguera, M.T. (2000). Estructura conductual en deportes sociomotores: Hockey sobre patines. Revista Digital (Buenos Aires), 21(5) [http://www.sportquest.com/revista/].

110. Hernández-Mendo, A. y Anguera, M.T. (2001). Estructura conductual en deportes sociomotores: Fútbol. Revista de Psicología Social, 16(1), 71-93.

111. Hernández-Mendo, A. y Planchuelo, L. (2013). El incremento del desarrollo moral en las clases de Educación Física. Revista Iberoamericana de Psicología del Ejercicio y el Deporte, 9(2), 369-392.

112. Hernández-Mendo, A., Castellano, J., Camerino, O., Jonsson, G., Blanco-Villaseńor, A., Lopes, A. y Anguera, M.T. (2014). Programas informáticos de registro, control de calidad del dato, y análisis de datos. Revista de Psicología del Deporte, 23(1), 111-121.

113. Hernández-Mendo, A., Díaz-Martínez, F., y Morales-Sánchez, V. (2010).Construcción de una herramienta observacional para evaluar las conductas prosociales en las clases de educación física. Revista de Psicología del Deporte, 19(2), 305-318.

114. Hernández-Mendo, A., López-López, J.A., Castellano, J., MoralesSánchez, V., Pastrana, J.L. (2012). HOISAN 1.2: Programa informático para uso en Metodología Observacional. Cuadernos de Psicología del Deporte, 12(1), 55-78.

115. Hernández-Mendo, A., Montoro, J., Reina, A. y Fernández-García, J.C. (2012). Desarrollo y optimización de una herramienta observacional para el bloqueo en voleibol. Revista Iberoamericana de Psicología del Ejercicio y el Deporte, 7(1), 15-32.

116. Hernández-Mendo, A., Ramos-Pérez, F., y Pastrana, J. L. (2012). SAGT: Programa informático para análisis de Teoría de la Generalizabilidad. SAFE CREATIVE Código: 1204191501059.

117. Hileno, R. y Buscà, B. (2012). Herramienta observacional para analizar la cobertura del ataque en voleibol. Revista Internacional de Medicina y Ciencias de la Actividad Física y el Deporte, 12(47), 557-570.

118. Holmes, P., Collins, D., \& Calmels, C. (2006). Electroencephalographic functional equivalence during observation of action. Journal of Sports Sciences, 24(6), 605-616.

119. Horn, R.R., Williams, M., \& Scott, M.A. (2002). Learning from demonstrations: The role of visual search during observationa learning from video and point-light models. Journal of Sports Sciences, 20, 253 269

120. Iglesias, X., Gasset, A., González, C., y Anguera, M.T. (2010). Interacción competitiva y presión ambiental en deportes de combate: aplicación de la metodología observacional. Revista Iberoamericana de Psicología del Deporte, 5(2), 267-282.

121. Iglesias, X., González, C. \& Anguera, M.T. (en prensa). Detección de regularidades en taekwondo de alto nivel. Cuadernos de Psicología del Deporte [en este número monográfico].

122. Iglesias, X., Rodríguez-Zamora, L., Chaverri, D., Rodríguez, F.A., Clapés, P. y Anguera, M.T. (en prensa). Diversificación de patrones en natación sincronizada de alto nivel. Cuadernos de Psicología del Deporte [en este número monográfico].

123. Iglesias, X., Rodríguez-Zamora, L., Clapés, P., Barrero, A., Chaverri, D., y Rodríguez, F.A. (2014). Análisis multidimensional de la estructura de las rutinas competitivas en natación sincronizada. Revista de Psicología del Deporte, 23(1), 173-180.

124. Ingebrigtsen, J., Dalen, T., Hjelde, G.H., Brust, B., \& WislØff, U. (2014). Acceleration and sprint profiles of a professional elite football team in match play. European Jorunal of Sport Science, http://dx.doi.or g/10.1080/17461391.2014.933879

125. Janssen, X., Cliff, D., Reilly, J., Hinkley, T., Jones, R., Batterham, M., Ekelund, U., Brage, S., \& Okely, T. (2014). Evaluation of Actical equations and thresholds to predict physical activity intensity in young children. Journal of Sports Sciences, http://dx.doi.org/10.1080/0264041 4.2014 .949826

126. Jonsson, G.K., Bjarkadottir, S.H., Gislason, B., Borrie, A. \& Magnus- 
son, M.S. (2003). Detection of real-time patterns in sports: interactions in football. L'éthologie appliquée aujourd'hui. In C. Baudoin (Ed.) Applied Ethology Today. Volume 3, Ethologie humaine. LevalloisPerret, France: Editions ED.

127. Jonsson, G., Blanco-Villaseńor, A., Losada, J.L. y Anguera, M.T. (2004). Avances en la codificación y análisis de eventos deportivos: Ilustración empírica en el fútbol. Metodología de las Ciencias del Comportamiento, vol. Especial, 317-322.

128. Jonsson, G.K., Anguera, M.T., Blanco-Villaseñor, A., Losada, J.L., Hernández-Mendo, A., Ardá, T., Camerino, O. \& Castellano, J. (2006). Hidden patterns of play interaction in soccer using SOF-CODER. Behavior Research Methods, 38(3), 372-381.

129. Jonsson, G.K., Anguera, M. T., Sánchez-Algarra, P., Oliveira, C., Campaniço, J., Castañer, M., Torrents, C., Chaverri, J., Camerino, O. \& Magnusson, M.S. (2010). Application of T-Pattern Detection and Analysis in Sports Research. The Open Sports Sciences Journal, 3, 62-71.

130. Lago, C. \& Anguera, M.T. (2002). Use of the polar coordinates technique to study interactions between professional soccer players. Revista Portuguesa de Ciências do Desporto, 2(4), 21-40.

131. Lago, C. y Anguera, M.T. (2003). Utilización del análisis secuencial en el estudio de las interacciones entre jugadores en el fútbol de rendimiento. Revista Española de Psicología del Deporte, 12(1), 27-37.

132. Lanzoni, I.M., Di Michele, R., \& Merni, F. (2014). A notational analysis of shot characteristics in top-level table tennis players. European Journal of Sport Science, 14(4), 309-317.

133. Lapresa, D., Alsasua, R., Arana, J., Anguera, M.T. y Garzón, B. (2014). Análisis observacional de la construcción de las secuencias ofensivas que acaban en lanzamiento en baloncesto de categoría infantil. Revista de Psicología del Deporte, 23(2), 365-376.

134. Lapresa, D., Álvarez, L., Arana, J., Garzón, B., \& Caballero, V. (2013). Observational analysis of the offensive sequences that ended in a shot by the winning team of the 2010 UEFA Futsal Championship. Journal of Sports Science, 31(5), 1731-1739.

135. Lapresa, D., Anguera, M.T., Alsasua, R., Arana, J. \& Garzón, B. (2013). Comparative analysis of T-patterns using real time data and simulated data by assignment of conventional durations: the construction of efficacy in children's basketball. International Journal of Performance Analysis in Sport, 13, 321-339.

136. Lapresa, D., Aragón. S. i Arana, J. (2012). Patrons temporals de comportament tàctic en curses atlétiques de 5.000 metres. Apunts. Educació Física i Esports, 109(3), 80-88.

137. Lapresa, D., Arana, J. i Garzón, B. (2006). El futbol 9 com alternativa al futbol 11, a partir de l'estudi de la utilització de l'espai de joc. Apunts. Educació Física i Esport, 86(4), 34-44.

138. Lapresa, D., Arana, J., Anguera, M.T. \& Garzón, B. (2013). Comparative analysis of the sequentiality using SDIS-GSEQ and THEME: a concrete example in soccer. Journal of Sports Sciences, 31(15), 1687-1695.

139. Lapresa, D., Arana, J., Anguera, M.T., Pérez, J.I. \& Amatria, M. (in press). Application of logistic regression models in observational methodology: game formats in grassroots football in initiation into football. Anales de Psicología.

140. Lapresa, D., Arana, J., Garzón, B., Egüén i Amatria, M. (2010). Adaptació de la competició en la iniciació al futbol: Estudi comparatiu de les modalitats de futbol 3 i futbol 5 en categoria prebenjamí. Apunts. Educació Física i Esport, 101(3), 43-56.

141. Lapresa, D., Camerino, O., Cabedo, J., Anguera, M.T., Jonsson, G., y Arana, J. (en prensa). Degradación de T-patterns en estudios observacionales: Un estudio sobre la eficacia en el ataque de fútbol sala. Cuadernos de Psicología del Deporte [en este número monográfico].

142. Lapresa, D., García Pascual, J., Arana, J., y Garzón, B. (2011). Análisis de patrones temporales en las rutinas gestuales previas al tiro libre de baloncesto, en la categoría alevín. Revista de Psicología del Deporte, 20(2), 383-400.
143. Lapresa, D., Ibáńez, R., Arana, J., Amatria, M. i Garzón, B. (2011). Estudi comparatiu de les accions de combat en el karate de categoria juvenil (12-13 anys) i sènior. Apunts. Educació Física i Esports, 104(2), 66-79.

144. Lees, A. (2002). Technique analysis in sports: a critical review. Journal of Sport Sciences, 20, 813-828.

145. Lonsdale, C. \& Tam, J.T.M. (2008). On the temporal and behavioural consistency of pre-performance routines: An intra-individual analysis of elite basketball players' free throw shooting accuracy. Journal of Sports Sciences, 26(3), 259-266.

146. López-López, J.A., Menescardi, C., Estevan, I., Falcó, C., y Hernández-Mendo, A. (en prensa). Análisis técnico-táctico en Taekwondo con coordenadas polares a través del software Hoisan. Cuadernos de Psicología del Deporte [en este número monográfico].

147. Lorenzo, J., Navarro, R., Rivilla, J. \& Lorenzo, A. (2013). The analysis of the basketball coach speech during the moments of game and pause in relation to the performance in competition. Revista de Psicología del Deporte, 22(1), 227-230.

148. Losada, J.L., Casal, C. y Ardá, T. (en prensa). Cómo mejorar la efectividad en un jugador de tenis: Modelos de regresión log-lineales. Cuadernos de Psicología del Deporte [en este número monográfico].

149. Louro, H., Silva, A. J., Anguera, M. T., Marinho, D. A., Oliveira, C., \& Campaniço, J. (2010). Stability of patterns of behavior in the butterfly technique of the elite swimmers. Journal of Sports Science and Medicine, 9, 36-50.

150. Magnusson, M.S. (1996). Hidden real-time patterns in intra- and inter-individual behavior. European Journal of Psychological Assessment, 12(2), 112-123.

151. Magnusson, M.S. (2000). Discovering hidden time patterns in behavior: T-patterns and their detection. Behavior Research Methods, Instruments, \& Computers, 32(1), 93-110.

152. Magnusson, M.S. (2005). Understanding social interaction: Discovering hidden structure with model and algorithms. In L. Anolli, S. Duncan, M. Magnusson \& G. Riva (Eds.), The hidden structure of social interaction. From Genomics to Culture Patterns (pp. 4-24). Amsterdam: IOS Press.

153. Magnusson, M.S. (2006). Structure and Communication in Interaction. In G. Riva, M.T. Anguera, B.K. Wiederhold \& F. Mantovani (Eds.), From Communication to Presence: Cognition, Emotions and Culture Towards the Ultimate Communicative Experience (pp. 127-146). Amsterdam: IOS Press.

154. Marcelino, R., Mesquita, I. \& Sampaio, J. (2011). Effects of quality of opposition and match status on technical and tactical performances in elite volleyball. Journal of Sports Sciences, 29(7), 733-741.

155. Marcelino, R., Mesquita, I., Sampaio, J. y Anguera, M.T. (2009). Ventaja de jugar em casa em voleibol de alto rendimiento. Revista de Psicología del Deporte, 18(2), 181-196.

156. Montoya, M., Moras, G. y Anguera, M.T. (2013). Análisis de las finalizaciones de los jugadores extremo en balonmano. Apunts. Educación Física y Deportes, 113 ( $3^{\text {er }}$ trimestre), 52-59.

157. Morales-Sánchez, V., Anguera, M.T., y Pérez-López, R. (2014). Tratamiento metodológico de la observación indirecta en la gestión de organizaciones deportivas. Revista de Psicología del Deporte, 23(1), 201-207.

158. Nadeau, L., Godbout, P. \& Richard, J.-F. (2008). Assessment of ice hockey performance in real-game conditions. European Journal of Sport Science, 8(6), 379-388.

159. Ollis, S., Macpherson, A. \& Collins, D. (2006). Expertise and talent development in rugby refereeing: An ethnographic enquiry. Journal of Sports Sciences, 24(3), 309-322.

160. Partington, M., Cushion, C., \& Harvey, S. (2014). An investigation of the effect of athletes' age on the coaching behaviours of professional top-level youth soccer coaches. Journal of Sports Sciences, 32(5), 403414. 
161. Peña, J., Rodríguez Guerra, J., Buscà, B. \& Serra, N. (2013). Which skills and factors better predict winning and losing in high-level men's volleyball? The Journal of Strength and Conditioning Research, 27(9), 2487-2493.

162. Pérez-López, R., Morales-Sanchez, V., Anguera, M.T. y HernándezMendo, A. (en prensa a). Evaluación de la calidad total en servicios municipales deportivos orientados a la población infantil: aportaciones desde el análisis cualitativo con Atlas ti. Cuadernos de Psicología del Deporte [en este número monográfico].

163. Pérez-López, R., Morales-Sánchez, V., Anguera, M.T. y HernándezMendo, A. (en prensa b). Hacia la calidad de servicio emocional en organizaciones deportivas orientadas a la población infantil: un análisis cualitativo. Revista Iberoamericana de Psicología del Ejercicio y el Deporte.

164. Planes, X. i Anguera, M.T. (en prensa). Rellevància dels diferents moments del joc ( $\mathrm{mj}$ ) i de les accions a balò parat ( $\mathrm{abp}$ ) en el rendiment del FC Barcelona i Reial Madrid durant la lliga nacional de futbol professional 2011/12. Apunts. Educació Física i Esports.

165. Prieto, I., Gutiérrez-Santiago, A., Camerino, O. \& Anguera, M.T. (2013). Knowledge of error in relation to the teaching and learning osoto-gari judo throw. International Journal of Sport Sciences \& Coaching, 8(1), 53-62.

166. Ramos, F. y Hernández-Mendo, A. (2014). Intervención para la reducción de la discriminación por sexo en las clases de Educación Física según los contenidos y agrupamientos utilizados. Revista Española de Educación Física y Deportes, 404(1), 27-38.

167. Reina, A., Hernández-Mendo, A. y Fernández-García, J. C. (2009). Multi-facet design for goal scoring in SOCCER-7. Quality \& Quantity, 44(5), 1025-1035.

168. Robles, J. F., y Castellano, J. (2012). Comparación entre el juego ofensivo de la selección espańola de fútbol y sus rivales en la Eurocopa'08 y Mundial'10. Revista Iberoamericana de Psicología del Ejercicio y el Deporte, $7(2), 307-322$.

169. Robles, F., Castellano, J., Perea, A., Martínez-Santos, R., \& Casamichana, D. (2013). Spatial strategy used by the world champion in south África'10. H. Nunome, B. Drust and D. Dawson (Ed.), Science and football VII (pp 161-168). London: Routledge.

170. Robles, J. F., Castellano, J. y Perea, A. (2014). Diferencias del juego entre la selección española de fútbol y sus rivales. Revista Iberoamericana de Ciencias de la Actividad Física y el Deporte, 3(2), 1-8.

171. Rubio, K. (2001). De la Psicología General a la Psicología del Deporte: Marcos teóricos, relaciones y dependencias. Revista de Psicología del Deporte, 10(2), 255-265.

172. Sackett, G.P. (1980) Lag sequential analysis as a data reduction technique in social interaction research. In D.B. Sawin, R.C. Hawkins, L.O. Walker \& J.H. Penticuff (Eds.). Exceptional infant. Psychosocial risks in infant-environment transactions (pp. 300-340). New York: Brunner/ Mazel.

173. Sackett, G.P. (Ed.)(1978). Observing Behavior (Vol. 2): Data collection and analysis methods. Baltimore: University of Park Press.

174. Sainz de Baranda, P. \& López-Riquelme, D. (2012). Analysis of corner kicks in relation to match status in the 2006 World Cup. European Journal of Sport Science, 12(2), 121-129.

175. Salas, C., Hileno, R., Molina, J.J. y Anguera, M.T. (2005). Análisis de la acción defensiva en voleibol: relación ataque- bloqueo. Kronos: la revista cientifica de actividad física y deporte, 8(62). Recuperado el 6 de Diciembre de 2013, desde http:/www.revistakronos.com/kronos/ index.php?articulo $=62$

176. Salas, C., Molina, J.J. y Anguera, M.T. (2008). Incidencia del número en la defensa de primera línea en voleibol. Apunts. Educació Física $i$ Esports, 93, 36-45.

177. Sánchez-Algarra, P. \& Anguera, M.T. (2013). Qualitative/quantitative integration in the inductive observational study of interactive behaviour: Impact of recording and coding predominating perspectives.
Quality \& Quantity. International Journal of Methodology, 47(2), 12371257.

178. Sánchez-Algarra, P. (Coord.) (2006). Métodos estadísticos aplicados. Barcelona: Publicacions i Edicions de la Universidad de Barcelona.

179. Santos, F.M., Fernandez, J., Oliveira, M.C., Leitão, C, Anguera, M.T. \& Campaniço, G. (2009). The pivot player in handball and patterns detection instrument. Motricidade, 5(3), 49-56.

180. Sarmento, H., Anguera, M.T., Pereira, A., Marques, A., Campaniço, J. \& Leitão, J. (2014). Patterns of play in the counterattack of elite football teams - A mixed method approach. International Journal of Performance Analysis in Sport, 14, 411-427.

181. Sarmento, H., Barbosa, A., Anguera, M.T., Campaniço, J. \& Leitão, J. (2013). Regular patterns of play in the counterattack of the FC Barcelona and Manchester United football teams. In D. Peters \& P. O'Donogue (Eds.), Performance Analysis of Sport IX (pp. 59-66). London: Routledge.

182. Sarmento, H., Pereira, A., Anguera, M.T., Campaniço, J., \& Leitão, A. (2014). The Coaching Process in Football - A qualitative perspective. Montenegrian Journal of Sports Sciences and Medicine, 3(1), 9-16.

183. Sarmento, H., Pereira, A., Campaniço, J., Anguera, M.T. \& Leitão, J. (2013). Soccer match analysis - Qualitative study with first Portuguese League coaches. In D. Peters \& P. O’Donogue (Eds.), Performance Analysis of Sport IX (pp. 10-16). London: Routledge.

184. Sarmento, H., Pereira, A., Matos, N., Campaniço, J., Anguera, M.T. \& Leitão, J. (2013). English Premier League, Spain's La Liga and Italy's Serie's A - What's different? International Journal of Sport Performance Analysis, 13, 773-789.

185. Sarmento, H., Pereira, A., Resende, R. \& Anguera, M.T. (2013). Padrôes de jogo no contra ataque das equipas do Barcelona FC, Inter de Miláo e Manchester United. Revista Mineira de Educação Fisica, 9 (Edição Especial), 508-514.

186. Sarmento, H., Marcelino, R., Anguera, M.T., Matos, N., Campaniço, J., \& Leitáo, J. (2014). Match analysis in football - A Systematic review. Journal of Sport Sciences, DOI: 10.1080/02640414.2014.898852

187. Saüch, G. y Castañer, M. (2014). Observación de patrones motrices generados por los programas de actividad física para la tercera edad y la percepción de sus usuarios. Revista de Psicología del Deporte, 23(1), 181-190.

188. Siegle, M. \& Lames, M. (2012). Game interruptions in elite soccer. Journal of Sports Sciences, 30(7), 619-624.

189. Silva, A., Sánchez Bańuelos, F., Garganta, J. y Anguera, M.T. (2005). Patrones de juego en el fútbol de alto rendimiento. Análisis secuencial del proceso ofensivo en el Campeonato del Mundo Corea-Japón 2002. Cultura, Ciencia y Deporte, 1(2), 65-72.

190. Smith, M. \& Cushion, C.J. (2006). An investigation of the in-game behaviours o professional, top-level youth soccer coaches. Journal of Sports Sciences, 24(4), 355-366.

191. Sousa, D., Prudente, J., Sequeira, P. y Hernández-Mendo, A. (en prensa). Ataque organizado en igualdad numérica en el Campeonato Europeo masculinos de Balonmano 2012. Analisis de Coordenadas Polares de las situaciones de 2 vs 2. Cuadernos de Psicología del Deporte [en este número monográfico].

192. Tarragó, R., Iglesias, X., Michavila, J., Chaverri, D., Ruiz, L. y Anguera, M.T. (en prensa). Análisis de patrones en asaltos de espada de alto nivel. Cuadernos de Psicología del Deporte [en este número monográfico].

193. Torrents, C. Castañer, M. \& Anguera, M.T. (2011). Dancing with complexity: Observation of emergent patterns in dance improvisation. Education, Physical Training, Sport, 80(1), 76-81.

194. Torrents, C. Castañer, M. Dinušová, M. \& Anguera, M.T. (2012). Dance divergently in physical education: The use of open-ended questions, metaphors, models and interaction with partners. Research in Dance Education, 13, 1-16. 
195. Torrents, C. Castañer, M. Dinušová, M. \& Anguera, M.T. (in press). Influence of the partner on motor skills generation dancing contact improvisation. International Journal of Sport Psychology. Special Issue 111.

196. Torrents, C., Castañer, M., Dinušová, M. \& Anguera, M.T. (2010). Discovering new ways of moving: Observational analysis of motor creativity while dancing contact improvisation and the influence of the partner. Journal of Creative Behavior, 44(1), 45-61.

197. Torrents, C., Castañer, M., Dinušová, M. y Anguera, M.T. (2008). El efecto del modelo docente y de la interacción con compañeros en las habilidades motrices creativas de la Danza. Un formato de campo para su análisis y obtención de T-patterns motrices. Retos. Nuevas tendencias en Educación Física, Deporte y Recreación, 14, 5-9.

198. Travassos, B., Duarte, R., Vilar, L., Davids, K. \& Araújo, D. (2012). Practice task design in team sports: Representativeness enhanced by increasing opportunities for action. Journal of Sports Sciences, 30(13), 1447-1454.

199. Triolet, C., Benguigui, N., Le Runigo, C. \& Williams, M. (2013). Quantifying the nature of anticipation in professional tennis. Journal of Sports Sciences, 31(8), 820-830.
200.Usabiaga, O., Castellano, J., Blanco-Villaseñor, A. y Casamichana, D. (2013). La Teoría de la Generalizabilidad en las primeras fases del método observacional aplicado en el ámbito de la iniciación deportiva: calidad del dato y estimación de la muestra. Revista de Psicología del Deporte, 22(1), 103-109.

201. Usabiaga, O., Castellano, J., y Blanco-Villaseńor, A. (2004). Precisión de generalización en un diseño multifaceta configurado para la observación de la pelota a mano por parejas. Metodología de las Ciencias del Comportamiento, volumen especial, 595-602.

202. Weinberg, R. S. y Gould, D. (1995). Foundations of sport and exercise psychology. Champaign: Human Kinetics.

203. Williams, J. M. y Straub, W. F. (1991). Nueva Psicología del Deporte: pasado, presente, futuro. En. J. M. Williams (Ed.), Psicología aplicada al deporte. Madrid: Biblioteca Nueva.

204.Zurloni, V., Cavalera, C., Diana, B., Elia, M., \& Jonsson, G.K. (2014). Detecting regularities in soccer dynamics: A T-pattern approach. Revista de Psicología del Deporte, 23(1), 157-164. 